Pacific

Journal of

Mathematics

GENERALIZED ELLIPTIC INTEGRALS AND MODULAR EQUATIONS

\author{
G.D. Anderson, S.-L. Qiu, M.K. Vamanamurthy, And M. \\ VUORINEN
}

Volume 192 No. 1

January 2000 


\title{
GENERALIZED ELLIPTIC INTEGRALS AND MODULAR EQUATIONS
}

\author{
G.D. Anderson, S.-L. Qiu, M.K. Vamanamurthy, and M. \\ VUORINEN
}

In geometric function theory, generalized elliptic integrals and functions arise from the Schwarz-Christoffel transformation of the upper half-plane onto a parallelogram and are naturally related to Gaussian hypergeometric functions. Certain combinations of these integrals also occur in analytic number theory in the study of Ramanujan's modular equations and approximations to $\pi$. The authors study the monotoneity and convexity properties of these quantities and obtain sharp inequalities for them.

\section{Introduction.}

In 1995 B. Berndt, S. Bhargava, and F. Garvan published an important paper $[\mathbf{B B G}]$ in which they studied generalized modular equations and gave proofs for numerous statements concerning these equations made by Ramanujan in his unpublished notebooks. No record of Ramanujan's original proofs has remained. A generalized modular equation with signature $1 / a$ and order (or degree) $p$ is

$$
\frac{F\left(a, 1-a ; 1 ; 1-s^{2}\right)}{F\left(a, 1-a ; 1 ; s^{2}\right)}=p \frac{F\left(a, 1-a ; 1 ; 1-r^{2}\right)}{F\left(a, 1-a ; 1 ; r^{2}\right)}, 0<r<1 .
$$

Here $F$ is the Gaussian hypergeometric function defined in (1.2). The word generalized alludes to the fact that the parameter $a \in(0,1)$ is arbitrary. In the classical case, $a=\frac{1}{2}$ and $p$ is a positive integer. Modular equations were studied extensively by Ramanujan, see [BBG], who also gave numerous algebraic identities for the solutions $s$ of (1.1) for some rational values of $a$ such as $\frac{1}{6}, \frac{1}{4}, \frac{1}{3}$.

Before proceeding, we introduce some necessary notation. Given complex numbers $a, b$, and $c$ with $c \neq 0,-1,-2, \ldots$, the Gaussian hypergeometric function is the analytic continuation to the slit plane $\mathbf{C} \backslash[1, \infty)$ of

$$
F(a, b ; c ; z)={ }_{2} F_{1}(a, b ; c ; z) \equiv \sum_{n=0}^{\infty} \frac{(a, n)(b, n)}{(c, n)} \frac{z^{n}}{n !}, \quad|z|<1 .
$$


Here $(a, 0)=1$ for $a \neq 0$, and $(a, n)$ is the shifted factorial function

$$
(a, n) \equiv a(a+1)(a+2) \cdots(a+n-1)
$$

for $n \in \mathbf{N} \equiv\{k: k$ is a positive integer $\}$. It is well known that $F(a, b ; c ; z)$ has many important applications, and many classes of special functions in mathematical physics are particular or limiting cases of this function. For these, and for properties of $F(a, b ; c ; z)$ see, for example, [AS, Ask, Be1, Be2, Be3, R, Var, WW].

To rewrite (1.1) in a slightly shorter form, we use the decreasing homeomorphism $\mu_{a}:(0,1) \rightarrow(0, \infty)$ defined by

$$
\mu_{a}(r) \equiv \frac{\pi}{2 \sin (\pi a)} \frac{F\left(a, 1-a ; 1 ; 1-r^{2}\right)}{F\left(a, 1-a ; 1 ; r^{2}\right)},
$$

for $a \in(0,1)$. We can now write (1.1) as

$$
\mu_{a}(s)=p \mu_{a}(r), \quad 0<r<1 .
$$

The solution of (1.4) is then given by

$$
s=\varphi_{K}^{a}(r) \equiv \mu_{a}^{-1}\left(\mu_{a}(r) / K\right), \quad p=1 / K .
$$

We call $\varphi_{K}^{a}(r)$ the modular function with signature $1 / a$ and degree $p=1 / K$.

For the parameter $K=1 / p$ with $p$ a small positive integer, the function (1.5) satisfies several algebraic identities. The main cases studied in $[\mathbf{B B G}]$ are:

$$
a=\frac{1}{6}, \frac{1}{4}, \frac{1}{3}, \quad p=2,3,5,7,11, \ldots .
$$

For generalized modular equations we use the Ramanujan notation:

$$
\alpha \equiv r^{2}, \quad \beta \equiv \varphi_{1 / p}^{a}(r)^{2} .
$$

We next state a few of the numerous identities $[\mathbf{B B G}]$ satisfied by $\varphi_{1 / p}^{a}(r)$ for various values of the parameters $a$ and $p$.

Theorem 1.6 ([BBG, Theorem 7.1]). If $\beta$ has degree 2 in the theory of signature 3 , then, with $a=\frac{1}{3}, \alpha=r^{2}, \beta=\varphi_{1 / 2}^{a}(r)^{2}$,

$$
(\alpha \beta)^{\frac{1}{3}}+\{(1-\alpha)(1-\beta)\}^{\frac{1}{3}}=1 .
$$

Theorem 1.7 ([BBG, Theorem 7.6]). If $\beta$ has degree 5 then, with $a=$ $\frac{1}{3}, \alpha=r^{2}, \beta=\varphi_{1 / 5}^{a}(r)^{2}$,

$$
(\alpha \beta)^{\frac{1}{3}}+\{(1-\alpha)(1-\beta)\}^{\frac{1}{3}}+3\{\alpha \beta(1-\alpha)(1-\beta)\}^{\frac{1}{6}}=1 .
$$

Theorem 1.8 ([BBG, Theorem 7.8]). If $\beta$ has degree 11 then, with $a=$ $\frac{1}{3}, \alpha=r^{2}, \beta=\varphi_{1 / 11}^{a}(r)^{2}$,

$$
\begin{aligned}
(\alpha \beta)^{\frac{1}{3}}+ & \{(1-\alpha)(1-\beta)\}^{\frac{1}{3}}+6\{\alpha \beta(1-\alpha)(1-\beta)\}^{\frac{1}{6}} \\
+ & 3 \sqrt{3}\{\alpha \beta(1-\alpha)(1-\beta)\}^{\frac{1}{12}}\left\{(\alpha \beta)^{\frac{1}{6}}+\{(1-\alpha)(1-\beta)\}^{\frac{1}{6}}\right\}=1
\end{aligned}
$$


Theorems 1.6-1.8 are surprising, because they provide algebraic identities for the modular function, which itself is defined in terms of the transcendental function $\mu_{a}(r)$. It is an interesting open problem to determine which of the modular equations in [BBG] can be solved algebraically, explicitly in terms of the modular function.

For $r \in(0,1), a \in(0,1)$, and $r^{\prime}=\sqrt{1-r^{2}}$, the generalized elliptic integrals (cf. [BB1, Section 5.5]) are defined by

$$
\left\{\begin{array}{l}
\mathcal{K}_{a}=\mathcal{K}_{a}(r)=\frac{\pi}{2} F\left(a, 1-a ; 1 ; r^{2}\right), \\
\mathcal{K}_{a}^{\prime}=\mathcal{K}_{a}^{\prime}(r) \equiv \mathcal{K}_{a}\left(r^{\prime}\right), \\
\mathcal{K}_{a}(0)=\frac{\pi}{2}, \mathcal{K}_{a}(1)=\infty,
\end{array}\right.
$$

and

$$
\left\{\begin{array}{l}
\mathcal{E}_{a}=\mathcal{E}_{a}(r) \equiv \frac{\pi}{2} F\left(a-1,1-a ; 1 ; r^{2}\right), \\
\mathcal{E}_{a}^{\prime}=\mathcal{E}_{a}^{\prime}(r) \equiv \mathcal{E}_{a}\left(r^{\prime}\right), \\
\mathcal{E}_{a}(0)=\frac{\pi}{2}, \mathcal{E}_{a}(1)=\frac{\sin (\pi a)}{2(1-a)} .
\end{array}\right.
$$

Clearly, $\mathcal{K}_{a}$ is increasing and $\mathcal{E}_{a}$ is decreasing on $(0,1)$. These functions satisfy the remarkable identity

$$
\mathcal{K}_{a}^{\prime} \mathcal{E}_{a}+\mathcal{K}_{a} \mathcal{E}_{a}^{\prime}-\mathcal{K}_{a} \mathcal{K}_{a}^{\prime}=\frac{\pi \sin (\pi a)}{4(1-a)},
$$

which is a special case of Elliott's formula (see (3.15)). This identity, along with some other properties of the functions $\mathcal{E}_{a}$ and $\mathcal{K}_{a}$, is very useful for the study of the function $\mu_{a}$. In the particular case $a=\frac{1}{2}$, the functions $\mathcal{K}_{a}(r)$ and $\mathcal{E}_{a}(r)$ reduce to $\mathcal{K}(r)$ and $\mathcal{E}(r)$, respectively, which are the well-known complete elliptic integrals of the first and second kind, respectively $[\mathbf{B o}, \mathbf{B F}]$. By symmetry of (1.9), unless stated otherwise, we assume that $a \in\left(0, \frac{1}{2}\right]$.

The purpose of this paper is to study the modular function $\varphi_{K}^{a}(r)$ for general $a \in\left(0, \frac{1}{2}\right]$, as well as the related functions $\mu_{a}, \mathcal{K}_{a}, \mathcal{E}_{a}, m_{a}$, and $\eta_{K}^{a}$, investigating their dependence on $r, K$, and $a$, where

$$
m_{a}(r) \equiv \frac{2}{\pi \sin (\pi a)} r^{2} \mathcal{K}_{a}(r) \mathcal{K}_{a}^{\prime}(r), \quad r \in(0,1),
$$

$$
\eta_{K}^{a}(x) \equiv\left(\frac{s}{s^{\prime}}\right)^{2}, \quad s=\varphi_{K}^{a}(r), r=\sqrt{\frac{x}{x+1}}, x, K \in(0, \infty) .
$$

For $a=\frac{1}{2}$ these functions reduce to well-known special cases denoted by $\varphi_{K}, \mu, \mathcal{K}, \stackrel{\mathcal{E}}{ }, m, \eta_{K}$, which often occur in geometric function theory $[\mathbf{A V V}$, 
$\mathbf{L V}]$, number theory [BB1], and analytic function theory. For example, $\mu(r)$ appears in the classical modular equation of degree $p, p>0$ (see [Be3] and [BB1]), that is, in the particular case $a=\frac{1}{2}$ of formula (1.1), while the upper bound in Schottky's Theorem is given in terms of $\mu(r)$ [Mart, Theorem 1.1]. Numerous properties of $\mu(r)$ have been studied (see, for instance, [AVV] and $[\mathbf{L V}])$. For $a=\frac{1}{3}$, J.M. Borwein and P.B. Borwein $[\mathbf{B B 2}]$ recently proved that there exists $\delta \in(0,1)$ such that the beautiful identity of Ramanujan,

$$
\mathcal{K}^{\prime}{ }_{1 / 3}\left(\left(\frac{1-r}{1+2 r}\right)^{3 / 2}\right)=(1+2 r) \mathcal{K}_{1 / 3}\left(r^{3 / 2}\right),
$$

is valid for all $r \in(0, \delta)$. They used it to derive a cubically convergent algorithm for the computation of $\pi$.

In Section 2 we construct a conformal map of a parallelogram with angles $\pi a, \pi(1-a), 0<a<1$, onto a half-plane. This map is denoted by $\mathrm{sn}_{a}$. For $a=\frac{1}{2}$ this map reduces to the well-known Jacobian elliptic function sn [Bo].

In Sections 3 and 4 we summarize some of the basic properties of the hypergeometric functions, obtaining a new proof for a formula due to Ramanujan. We also derive differentiation formulas for $\mathcal{K}_{a}, \mathcal{E}_{a}$, for applications in Section 5, in which we generalize several inequalities for $\mathcal{K}_{a}, \mathcal{E}_{a}, \mu_{a}$, proved in $[\mathbf{A V V}]$ for $a=\frac{1}{2}$. Our main results, which are based on the work in Section 5, are proved in Section 6.

We now state some of our main results for the generalized modular function $\varphi_{K}^{a}(r)$. The first two results show that this function satisfies simple multiplicative functional inequalities. For the special case $a=\frac{1}{2}$ see $[\mathbf{A V V}$, Lemma 10.7 and Theorem 10.28].

Theorem 1.14. For each $a \in\left(0, \frac{1}{2}\right]$ and $K \in(1, \infty)$, the function $f(x) \equiv$ $\log \left(1 / \varphi_{K}^{a}\left(e^{-x}\right)\right)$ is increasing and convex on $(0, \infty)$, while $g(x) \equiv$ $\log \left(1 / \varphi_{1 / K}^{a}\left(e^{-x}\right)\right)$ is increasing and concave on $(0, \infty)$. In particular,

$$
\begin{aligned}
\varphi_{K}^{a}(r) \varphi_{K}^{a}(t) & \leq\left(\varphi_{K}^{a}(\sqrt{r t})\right)^{2}, \\
\varphi_{1 / K}^{a}(r) \varphi_{1 / K}^{a}(t) & \geq\left(\varphi_{1 / K}^{a}(\sqrt{r t})\right)^{2} .
\end{aligned}
$$

Theorem 1.15. For each $a \in\left(0, \frac{1}{2}\right], K \in(1, \infty)$, and $r \in(0,1)$, the function $f(x) \equiv \varphi_{K}^{a}(r x) / \varphi_{K}^{a}(x)$ is increasing from $(0,1)$ onto $\left(r^{1 / K}, \varphi_{K}^{a}(r)\right)$, while $g(x) \equiv \varphi_{1 / K}^{a}(r x) / \varphi_{1 / K}^{a}(x)$ is decreasing from $(0,1)$ onto $\left(\varphi_{1 / K}^{a}(r), r^{K}\right)$. In particular,

$$
\begin{aligned}
\varphi_{K}^{a}(r t) & \leq \varphi_{K}^{a}(r) \varphi_{K}^{a}(t), \\
\varphi_{1 / K}^{a}(r t) & \geq \varphi_{1 / K}^{a}(r) \varphi_{1 / K}^{a}(t)
\end{aligned}
$$

for each $r, t \in(0,1)$, with equality if and only if $K=1$.

Because the derivative $\partial \varphi_{K}^{a}(r) / \partial r$ is unbounded on $(0,1)$, we now introduce a simple transformation which, when applied to $\varphi_{K}^{a}(r)$, yields a 
function whose derivative has range $(1 / K, K)$. Because of its moderating influence on $\varphi_{K}^{a}(r)$, we refer to this transformation as a linearization.

Theorem 1.16. Let $p:(0,1) \rightarrow(-\infty, \infty)$ and $q:(-\infty, \infty) \rightarrow(0,1)$, be given by $p(x)=2 \log \left(x / x^{\prime}\right)$ and $q(x)=p^{-1}(x)=\sqrt{e^{x} /\left(e^{x}+1\right)}$, and for $a \in\left(0, \frac{1}{2}\right], K \in(1, \infty)$ let $g, h:(-\infty, \infty) \rightarrow(-\infty, \infty)$ be defined by $g=p \circ \varphi_{K}^{a} \circ q, h=p \circ \varphi_{1 / K}^{a} \circ q$. Then $g$ and $h$ are increasing, $g$ is convex, $h$ is concave, and

$$
\begin{aligned}
& \frac{1}{K} \leq g^{\prime}(x) \leq K, \\
& \frac{1}{K} \leq h^{\prime}(x) \leq K,
\end{aligned}
$$

for all real $x$.

In Section 7 we study the dependence of the functions $\mathcal{K}_{a}, \mathcal{E}_{a}, \mu_{a}, \mu_{a}^{-1}$, and $\varphi_{K}^{a}$ on the parameter $a$.

Throughout this paper the hyperbolic sine, cosine, and tangent functions and their inverses are denoted by sh, ch, th and arsh, arch, arth, respectively. Whenever $r \in(0,1)$ we denote $r^{\prime}=\sqrt{1-r^{2}}$.

\section{Conformal mapping of a half plane onto a parallelogram.}

Definition 2.1. For each number $a \in\left(0, \frac{1}{2}\right], r \in(0,1)$, Im $t \geq 0$, let $g(t) \equiv$ $t^{-a}(1-t)^{a-1}\left(1-r^{2} t\right)^{-a}$ denote the analytic branch such that the argument of each of the factors $t, 1-t$, and $1-r^{2} t$, is $\pi$ whenever it is real and negative. We define the generalized elliptic sine function $\operatorname{sn}_{a}(w)=\operatorname{sn}_{a}(w, r)$ to be the inverse of the function

$$
w=f(z) \equiv \frac{\sin (\pi a)}{2} \int_{0}^{z} g(t) d t, \quad \operatorname{Im} z \geq 0 .
$$

By [Bo, p. 61, Example VI (4)], $f$ is a conformal mapping of the upper half $z$-plane onto the interior of a parallelogram with angles $\pi a$ and $\pi(1-a)$. The next result makes this notion more precise.

Theorem 2.2. Let $H$ denote the closed upper half-plane $\operatorname{Im} z \geq 0$, and let $r \in(0,1)$. The function $f$ defined above is a homeomorphism of $H$ onto the parallelogram with vertices $f(0)=0, f(1)=\mathcal{K}_{a}(r), f\left(1 / r^{2}\right)=$ $\mathcal{K}_{a}(r)+e^{i(1-a) \pi} \mathcal{K}_{a}^{\prime}(r)$, and $f(\infty)=e^{i(1-a) \pi} \mathcal{K}_{a}^{\prime}(r)$, conformal in the interior.

Proof. The function $f$ is a Schwarz-Christoffel mapping of $H$ onto the polygon with interior angles $(1-a) \pi, a \pi,(1-a) \pi$, and $a \pi$, hence a parallelogram [Mark, §20]. Clearly $f(0)=0$ and $f(1)=\mathcal{K}_{a}(r)$. Next, by [AVV, Theorem 1.19 (2)],

$$
f\left(\frac{1}{r^{2}}\right)=\frac{\sin (\pi a)}{2} \int_{0}^{1 / r^{2}} g(t) d t
$$




$$
\begin{aligned}
& =\frac{\sin (\pi a)}{2} \int_{0}^{1} g(t) d t+\frac{\sin (\pi a)}{2} \int_{1}^{1 / r^{2}} g(t) d t \\
& =\mathcal{K}_{a}(r)+\frac{\sin (\pi a)}{2} \int_{1}^{1 / r^{2}} g(t) d t \\
& =\mathcal{K}_{a}(r)+\frac{\sin (\pi a)}{2} \int_{0}^{1} e^{i \pi(1-a)} u^{a-1}(1-u)^{-a}\left(1-r^{\prime 2} u\right)^{a-1} d u \\
& =\mathcal{K}_{a}(r)+e^{i \pi(1-a)} \mathcal{K}_{a}^{\prime}(r),
\end{aligned}
$$

where in the last step we have made the change of variable $t=1 /\left(1-r^{\prime 2} u\right)$. Finally, by symmetry, $f(\infty)=e^{i \pi(1-a)} \mathcal{K}_{a}^{\prime}(r)$.

Corollary 2.3. Let $P$ be a parallelogram with sides of length $L, L^{\prime}$ and angles $\pi(1-a), \pi a, 0<a<\frac{1}{2}$. Then the conformal modulus of $P[\mathbf{L V}]$ is $\mathcal{K}^{\prime}(r) / \mathcal{K}(r)$, where

$$
r=r_{a} \equiv \mu_{a}^{-1}\left(\frac{\pi}{2 \sin (\pi a)} \frac{L^{\prime}}{L}\right)
$$

Proof. Choose $r$ so $\mathcal{K}_{a}^{\prime}(r) / \mathcal{K}_{a}(r)=L^{\prime} / L$. Then $P$ is similar to the parallelogram $P^{\prime}$ with vertices $0, \mathcal{K}_{a}(r), \mathcal{K}_{a}(r)+e^{i \pi(1-a)} \mathcal{K}_{a}^{\prime}(r)$, and $e^{i \pi(1-a)} \mathcal{K}_{a}^{\prime}(r)$. Then by (1.3) we have

$$
\mu_{a}(r)=\frac{\pi}{2 \sin (\pi a)} \frac{L^{\prime}}{L}
$$

implying that $r$ is as in (2.4). By Theorem 2.2, $\mathrm{sn}_{a}$ maps $P^{\prime}$ conformally onto the upper half-plane, taking its vertices onto $0,1,1 / r^{2}$, and $\infty$, respectively. Since the Jacobian elliptic sine function $\mathrm{sn}=\mathrm{sn}_{1 / 2}$ also maps the rectangle with vertices $0, \mathcal{K}(r), \mathcal{K}(r)+i \mathcal{K}^{\prime}(r)$, and $\mathcal{K}^{\prime}(r)$ conformally onto the upper half plane, taking its vertices onto $0,1,1 / r^{2}$, and $\infty$, respectively [Bo], it follows that the conformal modulus of $P$ is $\mathcal{K}^{\prime}(r) / \mathcal{K}(r)$.

Remark 2.5. Given two parallelograms $P$ and $Q$ with angles $\pi(1-a)$, $\pi a$ and $\pi(1-b), \pi b$, and sides of lengths $A, A^{\prime}$ and $B, B^{\prime}$, respectively, the extremal quasiconformal mapping with least dilatation $K$ from $P$ onto $Q$, is given by

$$
f=\psi \circ \mathrm{sn}_{b}^{-1}(\cdot, s) \circ \operatorname{sn}(\cdot, s) \circ g \circ \mathrm{sn}^{-1}(\cdot, r) \circ \operatorname{sn}_{a}(\cdot, r) \circ \varphi,
$$

where $\varphi$ and $\psi$ are similarity mappings and $g$ is an affine mapping. Here $r=\mu_{a}^{-1}\left(\frac{\pi}{2 \sin (\pi a)} \frac{A^{\prime}}{A}\right), s=\mu_{b}^{-1}\left(\frac{\pi}{2 \sin (\pi b)} \frac{B^{\prime}}{B}\right)$, and $K=\max \left\{\frac{\mu(r)}{\mu(s)}, \frac{\mu(s)}{\mu(r)}\right\}$. 


\section{Properties of $F(a, b ; c ; x)$.}

In this section, we study some monotoneity properties of the function $F(a, b ; c ; x)$ and certain of its combinations with other functions. We first recall some well-known properties of this function which will be used in the sequel.

It is well known that hypergeometric functions are closely related to the classical gamma function $\Gamma(x)$, the psi function $\psi(x)$, and the beta function $B(x, y)$. For $\operatorname{Re} x>0, \operatorname{Re} y>0$, these functions are defined by

$$
\Gamma(x) \equiv \int_{0}^{\infty} e^{-t} t^{x-1} d t, \psi(x) \equiv \frac{\Gamma^{\prime}(x)}{\Gamma(x)}, B(x, y) \equiv \frac{\Gamma(x) \Gamma(y)}{\Gamma(x+y)},
$$

respectively (cf. [WW]). It is well known that the gamma function satisfies the difference equation [WW, p. 237]

$$
\Gamma(x+1)=x \Gamma(x),
$$

and the reflection property $[\mathbf{W W}$, p. 239]

$$
\Gamma(x) \Gamma(1-x)=\frac{\pi}{\sin \pi x}=B(x, 1-x) .
$$

We shall also need the function

$$
R(a, b) \equiv-2 \gamma-\psi(a)-\psi(b), R(a) \equiv R(a, 1-a), \quad R\left(\frac{1}{2}\right)=\log 16,
$$

where $\gamma$ is the Euler-Mascheroni constant given by

$$
\gamma=\lim _{n \rightarrow \infty}\left(\sum_{k=1}^{n} \frac{1}{k}-\log n\right)=0.577215 \ldots
$$

By $[\mathbf{Q V u 2 , ~ L e m m a ~} 2.14(2)]$, for $a \in\left(0, \frac{1}{2}\right]$,

$$
R(a) \equiv R(a, 1-a) \geq A \cdot\left(\frac{1}{2}-a\right)^{2}+\log 16,
$$

with equality if and only if $a=\frac{1}{2}$, where $A=14 \cdot \zeta(3)=16.82879 \ldots$, and where $\zeta(\cdot)$ is the Riemann zeta function $[\mathbf{W W}]$.

The hypergeometric function (1.2) has the following simple differentiation formula

$$
\frac{d}{d x} F(a, b ; c ; x)=\frac{a b}{c} F(a+1, b+1 ; c+1 ; x) .
$$

An important tool for our work is the following classification of the behavior of the hypergeometric function near $x=1$ in the three cases 
$a+b<c, a+b=c$, and $a+b>c$ :

$$
\left\{\begin{array}{l}
F(a, b ; c ; 1)=\frac{\Gamma(c) \Gamma(c-a-b)}{\Gamma(c-a) \Gamma(c-b)}, a+b<c, \\
B(a, b) F(a, b ; a+b ; x)+\log (1-x)=R(a, b)+\mathrm{O}((1-x) \log (1-x)), \\
F(a, b ; c ; x)=(1-x)^{c-a-b} F(c-a, c-b ; c ; x), c<a+b .
\end{array}\right.
$$

The above asymptotic formula for the zero-balanced case $a+b=c$ is due to Ramanujan (see [Ask], [Be2]). This formula is implied by [AS, 15.3.10].

The asymptotic formula (3.8) gives us a precise description of the behavior of the function $F(a, b ; a+b ; x)$ near the logarithmic singularity $x=1$. In the next theorem we show that by an exponential change of variables we can cancel this singularity and that the transformed function will be nearly linear.

Theorem 3.9. For $a, b>0$, define $g(x)=F\left(a, b ; a+b ; 1-e^{-x}\right), x>0$. Then $g$ is an increasing and convex function with $g^{\prime}((0, \infty))=(a b /(a+b)$, $\Gamma(a+b) /(\Gamma(a) \Gamma(b)))$.

Proof. By [AS, 15.3.3] for $|z|<1$,

$$
F(a, b ; a+b+1 ; z)=(1-z) F(a+1, b+1 ; a+b+1 ; z) .
$$

From this relation and (3.7) we obtain

$$
\begin{aligned}
(a+b) g^{\prime}(x) /(a b) & =F\left(a+1, b+1 ; a+b+1 ; 1-e^{-x}\right) e^{-x} \\
& =F\left(a, b ; a+b+1 ; 1-e^{-x}\right),
\end{aligned}
$$

so that $g^{\prime}$ is positive and increasing and has the asserted range, by (3.8).

Theorem 3.10. Given $a, b>0$, and $a+b>c, d \equiv a+b-c$, the function $f(x)=F\left(a, b ; c ; 1-(1+x)^{-1 / d}\right), x>0$, is increasing and convex, with $f^{\prime}((0, \infty))=(a b /(c d), \Gamma(c) \Gamma(d) /(\Gamma(a) \Gamma(b)))$.

Proof. By [AS, 15.2.1 and 15.3.3],

$$
\begin{aligned}
f^{\prime}(x) & =\frac{a b}{c} F\left(a+1, b+1 ; c+1 ; 1-(1+x)^{-1 / d}\right)(1+x)^{-1 / d-1} \frac{1}{d} \\
& =\frac{a b}{c d} \frac{\left((1+x)^{-1 / d}\right)^{-d-1}}{(1+x)^{1+1 / d}} F\left(c-a, c-b ; c+1 ; 1-(1+x)^{-1 / d}\right) \\
& =\frac{a b}{c d} F\left(c-a, c-b ; c+1 ; 1-(1+x)^{-1 / d}\right),
\end{aligned}
$$

so that $f^{\prime}$ is increasing and has the asserted range, by (3.8). 
3.11. Gauss contiguous relations and derivative formula. The six functions $F(a \pm 1, b ; c ; z), F(a, b \pm 1 ; c ; z), F(a, b ; c \pm 1 ; z)$ are called contiguous to $F(a, b ; c ; z)$. Gauss gave 15 relations between $F(a, b ; c ; z)$ and pairs of its contiguous functions [AS, 15.2.10-15.2.27], [R, Section 33]. Using these relations, we shall write the differentiation formula (3.7) as in Theorem 3.12, which will be useful in our study. In particular, the differentiation formulas for the generalized elliptic integrals in Section 4 follow from Theorem 3.12. We prove this result, since this is not included in $[\mathbf{A S}]$ and since we have not found a proof in the literature. However, parts (1) and (2) of Theorem 3.12 are stated in [Mi, p. 267] in a slightly different form. Part (4) of Theorem 3.12 seems to be new.

Theorem 3.12. For $a, b, c>0, r \in(0,1)$, let $u=u(r)=F(a-1, b ; c ; r)$, $v=v(r)=F(a, b ; c ; r), u_{1}=u(1-r), v_{1}=v(1-r)$. Then

$$
\begin{gathered}
r \frac{d u}{d r}=(a-1)(v-u), \\
r(1-r) \frac{d v}{d r}=(c-a) u+(a-c+b r) v,
\end{gathered}
$$

or, equivalently,$$
\frac{a b}{c} r(1-r) F(a+1, b+1 ; c+1 ; r)=(c-a) u+(a-c+b r) v,
$$$$
\left\{\begin{array}{l}
r(1-r) \frac{d}{d r}\left(u v_{1}+u_{1} v-v v_{1}\right)= \\
(1-a-b)\left[(1-r) u v_{1}-r u_{1} v-(1-2 r) v v_{1}\right] .
\end{array}\right.
$$

Proof. (1) Observing that

$$
\begin{aligned}
\left(r \frac{d}{d r}+(a-1)\right) u(r) & =\sum_{n=0}^{\infty}(a-1+n) \frac{(a-1, n)(b, n)}{(c, n) n !} r^{n} \\
& =(a-1) \sum_{n=0}^{\infty} \frac{(a, n)(b, n)}{(c, n) n !} r^{n}=(a-1) v(r),
\end{aligned}
$$

we have the desired equality.

(2) If we change $a-1$ to $a$ in (1), then

$$
\frac{d v}{d r}=\frac{a}{r}(w-v)
$$

where $w=F(a+1, b ; c ; r)$. Gauss' relation for contiguous functions [AS, 15.2.10] yields

$$
w=\frac{1}{a(1-r)}((c-a) u+(2 a-c-a r+b r) v) .
$$


Thus

$$
\begin{aligned}
\frac{d v}{d r} & =\frac{a}{r}\left(\frac{1}{a(1-r)}((c-a) u+(2 a-c-a r+b r) v)-v\right) \\
& =\frac{(c-a)}{r(1-r)} u+\frac{(a-c+b r)}{r(1-r)} v,
\end{aligned}
$$

as desired.

Part (3) follows from (3.7) and part (2). Part (4) follows from (1), (2), and the product and chain rules.

We next apply Theorem 3.12 to give a new proof in Corollary 3.13 (3) for a special case of Ramanujan's formula for the derivative of the quotient of two hypergeometric functions (cf. [Be2, p. 88, Corollary]). In Corollary 3.13 (5) we give a generalization of formula (1.11). Both (1.11) and 3.13 (5) are generalizations of the Legendre relation for elliptic integrals.

Corollary 3.13. For $a, b, c>0, r \in(0,1)$, let $u=u(r)=F(a-1, b ; c ; r)$, $v=v(r)=F(a, b ; c ; r), u_{1}=u(1-r)$, and $v_{1}=v(1-r)$. Then

$$
-r(1-r) v^{2} \frac{d}{d r}\left(\frac{v_{1}}{v}\right)=(c-a)\left(u v_{1}+u_{1} v\right)+(b+2(a-c)) v v_{1}
$$

and

$$
r \frac{d}{d r}\left[(1-r) v v_{1}\right]=(c-a)\left[u v_{1}-u_{1} v\right]+[(2 b-1) r-b] v v_{1} .
$$

For $n=0,1,2, \ldots$,

$$
r \frac{d^{n+1} u}{d r^{n+1}}=(a-1) \frac{d^{n} v}{d r^{n}}-(a+n-1) \frac{d^{n} u}{d r^{n}},
$$

$r(1-r) \frac{d^{n+1} v}{d r^{n+1}}=(a-c+b r+2 n r-n) \frac{d^{n} v}{d r^{n}}+\left(n^{2}+n(b-1)\right) \frac{d^{n-1} v}{d r^{n-1}}+(c-a) \frac{d^{n} u}{d r^{n}}$.

In particular, if $a \in(0,1), b=1-a<c$, then

$$
u v_{1}+u_{1} v-v v_{1}=u(1)=\frac{\Gamma(c)^{2}}{\Gamma(c+a-1) \Gamma(c-a+1)} .
$$

In addition, if $c=1$, then

$$
\begin{gathered}
\frac{d}{d r}\left(\frac{v_{1}}{v}\right)=-\frac{\sin (\pi a)}{\pi r(1-r) v^{2}}, \\
r \frac{d}{d r}\left((1-r) v v_{1}\right)=\frac{\sin (\pi a)}{\pi}+v\left[(1-2 a) r v_{1}-2(1-a) u_{1}\right] .
\end{gathered}
$$


Proof. Parts (1)-(4) follow from Theorem 3.12 (1) and the Leibnitz Rule for higher derivatives. Next, let $a, b \in(0,1)$ and $b=1-a<c$. Then Theorem 3.12 (4) implies that the function in (5) is constant. If we let $r$ tend to 0 and make use of (3.7) and (3.8), it follows that this constant has the asserted value, and (5) follows. If, in addition, $c=1$, then (6) and (7) follow from (1) and (2), respectively.

3.14. Elliott's formula. E.B. Elliott [El] (cf. [Ba, p. 85]) proved the identity

$$
\begin{aligned}
& F\left(\frac{1}{2}+\lambda,-\frac{1}{2}-\nu ; 1+\lambda+\mu ; z\right) F\left(\frac{1}{2}-\lambda, \frac{1}{2}+\nu ; 1+\nu+\mu ; 1-z\right) \\
& +F\left(\frac{1}{2}+\lambda, \frac{1}{2}-\nu ; 1+\lambda+\mu ; z\right) F\left(-\frac{1}{2}-\lambda, \frac{1}{2}+\nu ; 1+\nu+\mu ; 1-z\right) \\
& -F\left(\frac{1}{2}+\lambda, \frac{1}{2}-\nu ; 1+\lambda+\mu ; z\right) F\left(\frac{1}{2}-\lambda, \frac{1}{2}+\nu ; 1+\nu+\mu ; 1-z\right) \\
& =\frac{\Gamma(1+\lambda+\mu) \Gamma(1+\nu+\mu)}{\Gamma\left(\lambda+\mu+\nu+\frac{3}{2}\right) \Gamma\left(\frac{1}{2}+\mu\right)} .
\end{aligned}
$$

If we put $\lambda=\nu=\frac{1}{2}-a$ and $\mu=c+a-\frac{3}{2}$ in Elliott's identity, we obtain the generalized Legendre relation in Corollary 3.13 (5), as was pointed out to us by B.C. Carlson.

Conjecture 3.16. Let $u(a, b, c, r)=F(a-1, b ; c ; r), v(a, b, c, r)=$ $F(a, b ; c ; r)$, and let

$$
\begin{gathered}
\mathcal{L}(a, b, c, r)=u(a, b, c, r) v(a, b, c, 1-r)+u(a, b, c, 1-r) v(a, b, c, r) \\
-v(a, b, c, r) v(a, b, c, 1-r)
\end{gathered}
$$

for $r \in(0,1)$. Note that $\mathcal{L}(a, a, a, r) \equiv 0$ for $a>0$. Observe also that by the generalized Legendre relation in Corollary $3.13(5), \mathcal{L}(a, 1-a, c, r)$ is a constant for $a \in(0,1), 1-a<c$. For $a, b \in(0,1), a+b \leq 1(\geq 1)$, we conjecture that the function $\mathcal{L}(a, b, 1, r)$ is concave (convex) as a function of $r$ on $(0,1)$.

\section{Derivative formulas.}

The following derivative formulas are analogous to those well-known ones when $a=\frac{1}{2}$. In particular, formulas (1), (2), (3), (4) are analogues of [BF, 710.00, 710.02, 710.05, 710.04], respectively, while (4), (5), (6), (7), (8), (9), (10), (11) generalize formulas (9), (19), (22), (23), (24), (18), (25), (26), respectively, in $[\mathbf{A V V}$, Appendix E]. 
Theorem 4.1. For each $a \in\left(0, \frac{1}{2}\right]$ the following derivative formulas hold for $r \in(0,1)$ and $x, y, K \in(0, \infty)$ :

$$
\begin{gathered}
\frac{\partial \varphi_{K}^{a}(r)}{\partial r}=\frac{1}{K} \frac{s s^{\prime 2} \mathcal{K}_{a}(s)^{2}}{r r^{\prime 2} \mathcal{K}_{a}(r)^{2}}=\frac{s s^{\prime 2} \mathcal{K}_{a}(s) \mathcal{K}_{a}^{\prime}(s)}{r r^{\prime 2} \mathcal{K}_{a}(r) \mathcal{K}_{a}^{\prime}(r)}=K \frac{s s^{\prime 2} \mathcal{K}_{a}^{\prime}(s)^{2}}{r r^{\prime 2} \mathcal{K}_{a}^{\prime}(r)^{2}} \\
\frac{\partial \varphi_{K}^{a}(r)}{\partial K}=\frac{4 s s^{\prime 2} \mathcal{K}_{a}(s)^{2}}{\pi^{2}} \frac{\mu_{a}(r)}{K^{2}}
\end{gathered}
$$

where $s=\varphi_{K}^{a}(r)$.

(9) $\frac{d m_{a}(r)}{d r}=\frac{2}{\pi r \sin (\pi a)}\left[\frac{\pi \sin (\pi a)}{2}-4(1-a) \mathcal{K}_{a}(r) \mathcal{E}_{a}^{\prime}(r)\right.$

$$
\left.+2(1-2 a) r^{2} \mathcal{K}_{a}(r) \mathcal{K}_{a}^{\prime}(r)\right]
$$

(10)

$$
\frac{\partial \eta_{K}^{a}(x)}{\partial x}=\frac{1}{K}\left(\frac{r^{\prime} s \mathcal{K}_{a}(s)}{r s^{\prime} \mathcal{K}_{a}(r)}\right)^{2}=K\left(\frac{r^{\prime} s \mathcal{K}_{a}^{\prime}(s)}{r s^{\prime} \mathcal{K}_{a}^{\prime}(r)}\right)^{2}=\left(\frac{r^{\prime} s}{r s^{\prime}}\right)^{2} \frac{\mathcal{K}_{a}(s) \mathcal{K}_{a}^{\prime}(s)}{\mathcal{K}_{a}(r) \mathcal{K}_{a}^{\prime}(r)} .
$$

$$
\frac{\partial \eta_{K}^{a}(x)}{\partial K}=\frac{8 \eta_{K}^{a}(x) \mu_{a}(r) \mathcal{K}_{a}(s)^{2}}{\pi^{2} K^{2}} .
$$

In (10) and (11), $r=\sqrt{x /(x+1)}$ and $s=\varphi_{K}^{a}(r)$. 
Proof. Formulas (1) and (2) follow from Theorem 3.12 and the Chain Rule (see also [BB1, (5.5.5)]), while (3) and (4) follow from (1) and (2). Formula (5) follows immediately from Corollary 3.13 (1), and (6) follows immediately from (5).

For (7), we let $s=\varphi_{K}^{a}(r)$. Then $\mu_{a}(s)=(1 / K) \mu_{a}(r)$. Hence, from (5),

$$
-\frac{\pi^{2}}{4 s s^{2} \mathcal{K}_{a}(s)^{2}} \frac{\partial s}{\partial r}=-\frac{1}{K} \frac{\pi^{2}}{4 r r^{\prime 2} \mathcal{K}_{a}(r)^{2}},
$$

and (7) follows. Similarly, from (5) we obtain

$$
-\frac{\pi^{2}}{4 s s^{\prime 2} \mathcal{K}_{a}(s)^{2}} \frac{\partial s}{\partial K}=-\frac{1}{K^{2}} \mu_{a}(r),
$$

which yields (8).

Formula (9) follows from Corollary 3.13 (7) and the Chain Rule.

For $(10)$, since $\eta_{K}^{a}(x)=\left(s / s^{\prime}\right)^{2}$, where $r=\sqrt{x /(x+1)}$ and $s=\varphi_{K}^{a}(r)$, formula (7) gives

$$
\begin{aligned}
\frac{\partial \eta_{K}^{a}(x)}{\partial x} & =2\left(\frac{s}{s^{\prime}}\right)^{2}\left[\frac{1}{s} \frac{\partial s}{\partial x}+\frac{s}{s^{\prime 2}} \frac{\partial s}{\partial x}\right]=2\left(\frac{s}{s^{\prime}}\right)^{2} \frac{1}{s s^{\prime 2}} \frac{1}{K} \frac{s s^{\prime 2} \mathcal{K}_{a}(s)^{2}}{r r^{\prime 2} \mathcal{K}_{a}(r)^{2}} \frac{\partial r}{\partial x} \\
& =2 \frac{s^{2}}{s^{\prime 2}} \frac{1}{K} \frac{\mathcal{K}_{a}(s)^{2}}{r r^{\prime 2} \mathcal{K}_{a}(r)^{2}} \frac{r^{\prime 4}}{2 r}=\frac{1}{K}\left(\frac{r^{\prime} s \mathcal{K}_{a}(s)}{r s^{\prime} \mathcal{K}_{a}(r)}\right)^{2} \\
& =K\left(\frac{r^{\prime} s \mathcal{K}_{a}^{\prime}(s)}{r s^{\prime} \mathcal{K}_{a}^{\prime}(r)}\right)^{2}=\left(\frac{r^{\prime} s}{r s^{\prime}}\right)^{2} \frac{\mathcal{K}_{a}(s) \mathcal{K}_{a}^{\prime}(s)}{\mathcal{K}_{a}(r) \mathcal{K}_{a}^{\prime}(r)} .
\end{aligned}
$$

Finally, for (11), with $\eta_{K}^{a}(x)=\left(s / s^{\prime}\right)^{2}$ as in (10), formula (8) yields

$$
\begin{aligned}
\frac{\partial \eta_{K}^{a}(x)}{\partial K} & =2\left(\frac{s}{s^{\prime}}\right)^{2}\left[\frac{1}{s}+\frac{s}{s^{\prime 2}}\right] \frac{\partial s}{\partial K} \\
& =2 \eta_{K}^{a}(x) \frac{1}{s s^{\prime 2}} \frac{4 s s^{\prime 2} \mathcal{K}_{a}(s)^{2}}{\pi^{2}} \frac{\mu_{a}(r)}{K^{2}} \\
& =\frac{8}{\pi^{2} K^{2}} \eta_{K}^{a}(x) \mu_{a}(r) \mathcal{K}_{a}(s)^{2} .
\end{aligned}
$$

4.2. Hypergeometric differential equation. Since the hypergeometric function $y=F(a, b ; c ; x)$ satisfies the differential equation

$$
x(1-x) y^{\prime \prime}+[c-(a+b+1) x] y^{\prime}-a b y=0
$$

$\left[\mathbf{R}\right.$, p. 54], it follows from the Chain Rule that $y=F\left(a, b ; c ; r^{2}\right)$ satisfies

$$
r r^{\prime 2} \frac{d^{2} y}{d r^{2}}+\left[(2 c-1)-(2 a+2 b+1) r^{2}\right] \frac{d y}{d r}-4 a b r y=0 .
$$


For a review of the theory of this differential equation, see [Var]. In particular, the functions $y=\mathcal{K}_{a}(r)\left(\right.$ or $\left.\mathcal{K}_{a}^{\prime}(r)\right)$ and $z=\mathcal{E}_{a}(r)\left(\right.$ or $\left.\mathcal{E}_{a}^{\prime}(r)\right)$ satisfy the differential equations

$$
\left\{\begin{array}{l}
r r^{\prime 2} \frac{d^{2} y}{d r^{2}}+\left(1-3 r^{2}\right) \frac{d y}{d r}-4 a(1-a) r y=0, \\
r r^{\prime 2} \frac{d^{2} z}{d r^{2}}+r^{\prime 2} \frac{d z}{d r}+4(1-a)^{2} r z=0 .
\end{array}\right.
$$

These reduce to the standard differential equations for $\mathcal{K}$ (or $\mathcal{K}^{\prime}$ ) and $\mathcal{E}$ (or $\left.\mathcal{E}^{\prime}\right)[\mathbf{B F}, 118.02]$, respectively, when $a=\frac{1}{2}$.

4.4. Particular values. According to [AS, 15.1.26], [BB1, p. 191, Exercise 22], or [R, p. 69, Exercise 3], we have the following formula:

$$
\mathcal{K}_{a}\left(\frac{1}{\sqrt{2}}\right)=\frac{c}{4 \sqrt{\pi}} \sin (\pi a), \quad c=\Gamma\left(\frac{1-a}{2}\right) \Gamma\left(\frac{a}{2}\right),
$$

while (4.5) and (1.11) give

$$
\mathcal{E}_{a}\left(\frac{1}{\sqrt{2}}\right)=\frac{4 \pi^{2}+(1-a) c^{2} \sin (\pi a)}{8 \sqrt{\pi}(1-a) c} .
$$

4.7. Some identities. It is easy to see that, for each $a \in\left(0, \frac{1}{2}\right]$, the function $\mu_{a}$ in (1.3) and its inverse are strictly decreasing functions and satisfy the identities

$$
\mu_{a}(r) \mu_{a}\left(r^{\prime}\right)=\frac{\pi^{2}}{4 \sin ^{2}(\pi a)}
$$

and

$$
\mu_{a}^{-1}(x)^{2}+\mu_{a}^{-1}(y)^{2}=1
$$

for $r \in(0,1), r^{\prime}=\sqrt{1-r^{2}}, x, y \in(0, \infty)$ with $x y=\pi^{2} /\left(4 \sin ^{2}(\pi a)\right)$ (see the solution of [AVV, Exercise 5.45 (1), p. 364]).

For $a=\frac{1}{2}$ the relation (4.8) reduces to a well-known property of the function $\mu(r)$ (cf. [LV, p. 61, (2.7)]). The function $m_{a}(r)$ introduced in (1.12) is easily seen to satisfy the identities

(4.10) $m_{a}(r)+m_{a}\left(r^{\prime}\right)=\frac{2}{\pi \sin (\pi a)} \mathcal{K}_{a}(r) \mathcal{K}_{a}^{\prime}(r)$ and $r^{2} m_{a}(r)=r^{\prime 2} m_{a}\left(r^{\prime}\right)$.

\section{Generalized elliptic integrals.}

The following monotone form of l'Hôpital's Rule [AVV, Theorem 1.25] will be extremely useful in our proofs. We have recently learned from R. Kellerhals of a similar result due to M. Gromov which is a handy tool for volume estimation in Riemannian geometry (see [C, p. 124, Lemma 3.1]). 
Lemma 5.1. For $-\infty<a<b<\infty$, let $f, g:[a, b] \rightarrow \mathbf{R}$ be continuous on $[a, b]$, and be differentiable on $(a, b)$. Let $g^{\prime}(x) \neq 0$ on $(a, b)$. If $f^{\prime}(x) / g^{\prime}(x)$ is increasing (decreasing) on $(a, b)$, then so are

$$
[f(x)-f(a)] /[g(x)-g(a)] \quad \text { and } \quad[f(x)-f(b)] /[g(x)-g(b)] .
$$

If $f^{\prime}(x) / g^{\prime}(x)$ is strictly monotone, then the monotoneity in the conclusion is also strict.

In the next two lemmas and the following theorem we study how these generalized elliptic integrals $\mathcal{K}_{a}$ and $\mathcal{E}_{a}$ depend upon the variable $r$. When $a=\frac{1}{2}$, Lemma $5.2(1),(3),(4),(10)$, (12) reduce to Theorem 3.21 (1), Exercise 3.43 (32), (46), and Theorem 3.31 (6), Exercise 3.43 (15), respectively, in $[\mathbf{A V V}]$.

Lemma 5.2. Let $a \in\left(0, \frac{1}{2}\right]$ be given, and let $b=1-a, c=(\sin (\pi a)) / b$. Then the function

(1) $f_{1}(r) \equiv\left(\mathcal{E}_{a}-r^{\prime 2} \mathcal{K}_{a}\right) / r^{2}$ is increasing and convex from $(0,1)$ onto $(\pi a / 2, c / 2)$.

(2) $f_{2}(r) \equiv r^{\prime 2} \mathcal{K}_{a} / \mathcal{E}_{a}$ is decreasing from $(0,1)$ onto $(0,1)$.

(3) $f_{3}(r) \equiv\left(\mathcal{K}_{a}-\mathcal{E}_{a}\right) /\left(r^{2} \mathcal{K}_{a}\right)$ is increasing from $(0,1)$ onto $(b, 1)$.

(4) $f_{4}(r) \equiv\left(\mathcal{E}_{a}-r^{2} \mathcal{K}_{a}\right) /\left(r^{2} \mathcal{K}_{a}\right)$ is decreasing from $(0,1)$ onto $(0, a)$.

(5) $f_{5}(r) \equiv\left(\mathcal{E}_{a}-r^{\prime 2} \mathcal{K}_{a}\right) /\left(\mathcal{K}_{a}-\mathcal{E}_{a}\right)$ is decreasing from $(0,1)$ onto $(0, a / b)$.

(6) $f_{6}(r) \equiv r^{\prime 2}\left(\mathcal{K}_{a}-\mathcal{E}_{a}\right) /\left(r^{2} \mathcal{E}_{a}\right)$ is decreasing from $(0,1)$ onto $(0, b)$.

(7) $f_{7}(r) \equiv\left((\pi / 2)^{2}-\left(r^{\prime} \mathcal{K}_{a}\right)^{2}\right) /\left(\mathcal{E}_{a}-r^{\prime 2} \mathcal{K}_{a}\right)$ is increasing from $(0,1)$ onto $\left(\pi\left(a^{2}+b^{2}\right) /(2 a), \pi^{2} /(2 c)\right)$.

(8) $f_{8}(r) \equiv\left(\mathcal{K}_{a}-\pi / 2\right) / \log \left(1 / r^{\prime}\right)$ is increasing from $(0,1)$ onto $(\pi a b, b c)$.

(9) $f_{9}(r) \equiv\left(\mathcal{E}_{a}-(1-r) \mathcal{K}_{a}\right) / r$ is decreasing from $(0,1)$ onto $(c / 2, \pi / 2)$.

(10) $f_{10}(r) \equiv r^{-2}\left[\left(\mathcal{E}_{a}-r^{\prime 2} \mathcal{K}_{a}\right) / r^{2}-\pi a / 2\right]$ is increasing and convex from $(0,1)$ onto $\left(\pi a^{2} b / 4,(c-\pi a) / 2\right)$.

(11) $f_{11}(r) \equiv\left[a\left(\mathcal{K}_{a}-\mathcal{E}_{a}\right)-(1-a)\left(\mathcal{E}_{a}-r^{\prime 2} \mathcal{K}_{a}\right)\right] /\left(2 \log \left(1 / r^{\prime}\right)-r^{2}\right)$ is increasing from $(0,1)$ onto $\left(\pi a^{2} b / 2, a b c / 2\right)$.

(12) $f_{12}(r) \equiv\left(\mathcal{K}_{a}-\mathcal{E}_{a}\right) / \log \left(1 / r^{\prime}\right)$ is decreasing from $(0,1)$ onto $(\sin (\pi a)$, $\pi b)$.

(13) $f_{13}(r) \equiv\left(\pi / 2-r^{\prime 2} \mathcal{K}_{a}\right) / r^{2}$ is increasing and convex from $(0,1)$ onto $\left(\pi\left(a^{2}+b\right) / 2, \pi / 2\right)$.

(14) $f_{14}(r) \equiv\left(\pi^{2} / 4-r^{2} \mathcal{K}_{a}^{2}\right) / r^{2}$ is increasing from $(0,1)$ onto $\left(\pi^{2}\left(a^{2}+b^{2}\right) / 4, \pi^{2} / 4\right)$.

Proof. (1) It follows from (1.2), (1.9), and (1.10) that 


$$
\begin{aligned}
\mathcal{E}_{a} & -r^{\prime 2} \mathcal{K}_{a} \\
= & \frac{\pi}{2}\left\{\sum_{n=0}^{\infty} \frac{(a-1)(a, n-1)(1-a, n)-(a, n)(1-a, n)}{(n !)^{2}} r^{2 n}\right. \\
& \left.+\sum_{n=0}^{\infty} \frac{(a, n)(1-a, n)}{(n !)^{2}} r^{2(n+1)}\right\} \\
= & \frac{\pi}{2} \sum_{n=1}^{\infty} \frac{n^{2}(1-a, n-1)(a, n-1)-(1-a, n)[(1-a)(1, n-1)+(a, n)]}{(n !)^{2}} r^{2 n} \\
= & \frac{\pi a}{2} \sum_{n=1}^{\infty} \frac{n(a, n-1)(1-a, n-1)}{(n !)^{2}} r^{2 n} \\
= & \frac{\pi a}{2} r^{2} \sum_{n=0}^{\infty} \frac{1}{n+1} a_{n} r^{2 n},
\end{aligned}
$$

where $a_{n}=(a, n)(1-a, n)(n !)^{-2}$, and hence the result follows immediately.

(2) This follows from $r^{\prime 2} \mathcal{K}_{a} / \mathcal{E}_{a}=1-\left(\left(\mathcal{E}_{a}-r^{\prime 2} \mathcal{K}_{a}\right) / \mathcal{E}_{a}\right)$, since $\mathcal{E}_{a}-r^{\prime 2} \mathcal{K}_{a}$ is increasing and $\mathcal{E}_{a}$ is decreasing by Theorem $4.1(4),(2)$, respectively. The limiting values are clear, as in (1).

(3) We write $f_{3}(r)=g_{3}(r) / h_{3}(r)$, where $g_{3}(r)=\mathcal{K}_{a}-\mathcal{E}_{a}$ and $h_{3}(r)=$ $r^{2} \mathcal{K}_{a}$. Then $g_{3}(0)=h_{3}(0)=0$ and

$$
\begin{aligned}
\frac{g_{3}^{\prime}(r)}{h_{3}^{\prime}(r)} & =\frac{2(1-a) r \mathcal{E}_{a}}{r^{\prime 2}\left[2 r \mathcal{K}_{a}+2(1-a) r\left(\mathcal{E}_{a}-r^{\prime 2} \mathcal{K}_{a}\right) / r^{\prime 2}\right]} \\
& =\frac{(1-a) \mathcal{E}_{a}}{(1-a) \mathcal{E}_{a}+a r^{\prime 2} \mathcal{K}_{a}}=\frac{1-a}{1-a+a r^{\prime 2} \mathcal{K}_{a} / \mathcal{E}_{a}},
\end{aligned}
$$

which is increasing by (2). Clearly $f_{3}(1-)=1$, while $f_{3}(0+)=1-a$ by l'Hôpital's Rule. Hence (3) follows from Lemma 5.1.

(4) This follows from (3), since $f_{4}(r)=1-f_{3}(r)$.

(5) This follows from (3) and (4).

(6) Since $f_{6}(r)=1-f_{1}(r) / \mathcal{E}_{a}$, the result follows from (1).

(7) Write $f_{7}(r)=g_{7}(r) / h_{7}(r)$, where $g_{7}(r)=\left(\pi^{2} / 4\right)-\left(r^{\prime} \mathcal{K}_{a}\right)^{2}$ and $h_{7}(r)=$ $\mathcal{E}_{a}-r^{\prime 2} \mathcal{K}_{a}$. Then $g_{7}(0)=h_{7}(0)=0$ and

$$
\begin{aligned}
\frac{g_{7}^{\prime}(r)}{h_{7}^{\prime}(r)} & =\frac{2 r \mathcal{K}_{a}^{2}-4(1-a) \mathcal{K}_{a}\left(\mathcal{E}_{a}-r^{\prime 2} \mathcal{K}_{a}\right) / r}{2 a r \mathcal{K}_{a}} \\
& =\frac{\mathcal{K}_{a}}{a}\left[1-2(1-a) \frac{\mathcal{E}_{a}-r^{\prime 2} \mathcal{K}_{a}}{r^{2} \mathcal{K}_{a}}\right]
\end{aligned}
$$


which is increasing by (4). By l'Hôpital's Rule, $f_{7}(0+)=\pi(1-2 a+$ $\left.2 a^{2}\right) /(2 a)$, while $f_{7}(1-)=\pi^{2} /\left(4 \mathcal{E}_{a}(1)\right)=\pi^{2}(1-a) /(2 \sin (\pi a))$.

(8) Write $f_{8}(r)=g_{8}(r) / h_{8}(r)$, where $g_{8}(r)=\mathcal{K}_{a}-\pi / 2$ and $h_{8}(r)=$ $\log \left(1 / r^{\prime}\right)$. Then $g_{8}(0)=h_{8}(0)=0$ and $g_{8}^{\prime}(r) / h_{8}^{\prime}(r)=2(1-a)\left(\mathcal{E}_{a}-r^{\prime 2} \mathcal{K}_{a}\right) / r^{2}$, which is increasing by (1). Hence $f_{8}(r)$ is increasing by Lemma 5.1. The limits $f_{8}(0+)=a(1-a) \pi$ and $f_{8}(1-)=2(1-a) \mathcal{E}_{a}(1)=\sin (\pi a)$ follow by l'Hôpital's Rule.

(9) Write $f_{9}(r)=g_{9}(r) / h_{9}(r)$, where $g_{9}(r)=\mathcal{E}_{a}-(1-r) \mathcal{K}_{a}$ and $h_{9}(r)=r$. Then $g_{9}(0)=h_{9}(0)=0$ and

$$
\begin{aligned}
\frac{g_{9}^{\prime}(r)}{h_{9}^{\prime}(r)} & =-2(1-a) \frac{\mathcal{K}_{a}-\mathcal{E}_{a}}{r}+\mathcal{K}_{a}-\frac{2(1-a)\left(\mathcal{E}_{a}-r^{2} \mathcal{K}_{a}\right)}{r(1+r)} \\
& =-(1-2 a) \mathcal{K}_{a}+2(1-a) \frac{\mathcal{E}_{a}}{1+r}
\end{aligned}
$$

which is decreasing. The limit $f_{9}(0+)$ follows from l'Hôpital's Rule, while $f_{9}(1-)$ is clear from (1.9). Hence $f_{9}$ is decreasing by Lemma 5.1.

(10) Since

$$
f_{10}(r)=\frac{\pi a}{2} \sum_{n=1}^{\infty} \frac{a_{n}}{n+1} r^{2(n-1)}
$$

by (5.3), the result follows immediately.

(11) Write $f_{11}(r)=g_{11}(r) / h_{11}(r)$, where $g_{11}(r)$ and $h_{11}(r)$ are the numerator and denominator, respectively, of the fraction defining $f_{11}(r)$. Then $g_{11}(0)=h_{11}(0)=0$ and

$$
\frac{g_{11}^{\prime}(r)}{h_{11}^{\prime}(r)}=a(1-a) \frac{\mathcal{E}_{a}-r^{\prime 2} \mathcal{K}_{a}}{r^{2}},
$$

which is increasing by (1), with limits $\pi a^{2}(1-a) / 2$ and $a(\sin (\pi a)) / 2$ at 0 and 1 , respectively. Hence the result follows from Lemma 5.1.

(12) Write $f_{12}(r)=g_{12}(r) / h_{12}(r)$, where $g_{12}(r)=\mathcal{K}_{a}-\mathcal{E}_{a}$ and $h_{12}(r)=$ $\log \left(1 / r^{\prime}\right)$. Then $g_{12}(0)=h_{12}(0)=0$ and $g_{12}^{\prime}(r) / h_{12}^{\prime}(r)=2(1-a) \mathcal{E}_{a}$, and the result follows from Lemma 5.1 since $\mathcal{E}_{a}$ is decreasing.

(13) It follows from (1.2) and (1.9) that

$$
\begin{aligned}
f_{13}(r) & =\frac{\pi}{2 r^{2}}\left[\sum_{n=0}^{\infty} \frac{(a, n)(1-a, n)}{(n !)^{2}} r^{2(n+1)}-\sum_{n=1}^{\infty} \frac{(a, n)(1-a, n)}{(n !)^{2}} r^{2 n}\right] \\
& =\frac{\pi}{2 r^{2}} \sum_{n=1}^{\infty} \frac{(a, n-1)(1-a, n-1)}{(n !)^{2}}\left(n+a^{2}-a\right) r^{2 n} \\
& =\frac{\pi}{2} \sum_{n=0}^{\infty} \frac{(a, n)(1-a, n)}{[(n+1) !]^{2}}\left(n+a^{2}+b\right) r^{2 n},
\end{aligned}
$$


and hence the result follows.

(14) Write $f_{14}(r)=g_{14}(r) / h_{14}(r)$, where $g_{14}(r)=\pi^{2} / 4-r^{\prime 2} \mathcal{K}_{a}^{2}$ and $h_{14}(r)=r^{2}$. Then $g_{14}(0)=h_{14}(0)=0$, and

$$
\frac{g_{14}^{\prime}(r)}{h_{14}^{\prime}(r)}=\mathcal{K}_{a}^{2} \cdot\left[1-\frac{2(1-a)\left(\mathcal{E}_{a}-r^{\prime 2} \mathcal{K}_{a}\right)}{r^{2} \mathcal{K}_{a}}\right]
$$

which is increasing, with $f_{14}(0+)=\pi^{2}\left(1-2 a+2 a^{2}\right) / 4$, by part (4). The value $f_{14}(1-)=\pi^{2} / 4$ is clear. Hence, the result follows from Lemma 5.1.

In the next lemma, when $a=\frac{1}{2}$, parts (1), (2), (4), (5) reduce to Theorem 3.21 (7), (8) and Exercise 3.43 (30), (45), respectively, in [AVV], while part (3) becomes the monotone property of $\mathcal{E}$.

Lemma 5.4. Let $a \in\left(0, \frac{1}{2}\right]$. Then the function

(1) $f_{1}(r) \equiv r^{\prime c} \mathcal{K}_{a}$ is decreasing if and only if $c \geq 2 a(1-a)$, in which case $r^{\prime c} \mathcal{K}_{a}$ is decreasing from $(0,1)$ onto $(0, \pi / 2)$. Moreover, $\sqrt{r^{\prime}} \mathcal{K}_{a}$ is decreasing for each $a \in\left(0, \frac{1}{2}\right]$.

(2) $f_{2}(r) \equiv r^{\prime} \mathcal{E}_{a}$ is increasing if and only if $c \leq-2(1-a)^{2}$. In particular, $r^{\prime-2(1-a)^{2}} \mathcal{E}_{a}$ is increasing from $(0,1)$ onto $(\pi / 2, \infty)$. Moreover, $\mathcal{E}_{a} / r^{\prime 2}$ is increasing for each $a \in\left(0, \frac{1}{2}\right]$.

(3) $f_{3}(r) \equiv \mathcal{E}_{a}+(1-2 a)\left(\mathcal{E}_{a}-r^{\prime 2} \mathcal{K}_{a}\right)$ is decreasing from $(0,1)$ onto $(\sin (\pi a), \pi / 2)$.

(4) $f_{4}(r) \equiv r \mathcal{K}_{a} /$ arth $r$ is decreasing from $(0,1)$ onto $(\sin (\pi a), \pi / 2)$.

(5) For $b=(\sin (\pi a)) /(2(1-a))$ and $0<c \leq 2\left(1-a+a^{2}\right)$, the function $f_{5, c}(r) \equiv\left(\mathcal{E}_{a}-r^{\prime 2} \mathcal{K}_{a}\right) /\left(1-r^{\prime c}\right)$ is decreasing from $(0,1)$ onto $(b, \pi a / c)$. In particular, $f_{5,3 / 2}$ is decreasing from $(0,1)$ onto $(b, 2 \pi a / 3)$.

Proof. (1) By Theorem 4.1 (1),

$$
r r^{\prime 2-c} f_{1}^{\prime}(r)=-c r^{2} \mathcal{K}_{a}+2(1-a)\left(\mathcal{E}_{a}-r^{\prime 2} \mathcal{K}_{a}\right),
$$

which is nonpositive if and only if

$$
c \geq 2(1-a) \sup _{r} \frac{\mathcal{E}_{a}-r^{\prime 2} \mathcal{K}_{a}}{r^{2} \mathcal{K}_{a}}=2 a(1-a),
$$

by Lemma $5.2(4)$. Finally, we note that since $\max \left\{2 a(1-a): 0<a \leq \frac{1}{2}\right\}=$ $\frac{1}{2}$, the function $\sqrt{r^{\prime}} \mathcal{K}_{a}$ will be decreasing for each $a \in\left(0, \frac{1}{2}\right]$. The limiting value at $r=0$ is obvious, while that at $r=1$ follows from l'Hôpital's Rule, Theorem 4.1 (1), and Lemma 5.2 (1).

(2) By Theorem 4.1 (2), we have

$$
r f_{2}^{\prime}(r)=-c r^{\prime c-2} r^{2} \mathcal{E}_{a}+2(a-1) r^{\prime c}\left(\mathcal{K}_{a}-\mathcal{E}_{a}\right),
$$

which is nonnegative if and only if

$$
-c \geq 2(1-a) \sup _{r} \frac{r^{\prime 2}\left(\mathcal{K}_{a}-\mathcal{E}_{a}\right)}{r^{2} \mathcal{E}_{a}}=2(1-a)^{2},
$$


by Lemma $5.2(6)$. Finally, since $\max \left\{2(1-a)^{2}: 0<a \leq \frac{1}{2}\right\}=2$, it follows that $\mathcal{E}_{a} / r^{\prime 2}$ will be increasing for each $a \in\left(0, \frac{1}{2}\right]$. The limiting values are clear from (1.9).

For (3) we may write

$$
r f_{3}^{\prime}(r)=-2(1-a)\left(\mathcal{K}_{a}-\mathcal{E}_{a}\right)+2 a(1-2 a) r^{2} \mathcal{K}_{a} .
$$

Hence, by Lemma $5.2(4)$,

$$
\frac{f_{3}^{\prime}(r)}{2 r \mathcal{K}_{a}}=2 a(1-a)-1+(1-a) \frac{\mathcal{E}_{a}-r^{\prime 2} \mathcal{K}_{a}}{r^{2} \mathcal{K}_{a}}<3 a(1-a)-1 \leq-\frac{1}{4},
$$

and so $f_{3}$ is decreasing on $(0,1)$. The limiting values are clear.

(4) Write $f_{4}(r)=g_{4}(r) / h_{4}(r)$, where $g_{4}(r)=r \mathcal{K}_{a}$ and $h_{4}(r)=\operatorname{arth} r$. Then $g_{4}(0)=h_{4}(0)=0$ and $g_{4}^{\prime}(r) / h_{4}^{\prime}(r)=f_{3}(r)$, hence decreasing by (3). By l'Hôpital's Rule, we have $f_{4}(0+)=\mathcal{K}_{a}(0)=\pi / 2$ and

$$
f_{4}(1-)=\lim _{r \rightarrow 1-}\left[2(1-a)\left(\mathcal{E}_{a}-r^{2} \mathcal{K}_{a}\right)\right]=2(1-a) \mathcal{E}_{a}(1)=\sin (\pi a) .
$$

(5) The limit $f_{5, c}(1-)$ is clear by (1) and (1.9). For monotoneity write $f_{5, c}(r)=g_{5}(r) / h_{5}(r)$, where $g_{5}(r)=\mathcal{E}_{a}-r^{\prime 2} \mathcal{K}_{a}$ and $h_{5}(r)=1-r^{\prime c}$. Then $g_{5}(0)=h_{5}(0)=0$ and

$$
\frac{g_{5}^{\prime}(r)}{h_{5}^{\prime}(r)}=\frac{2 a}{c} r^{\prime 2-c} \mathcal{K}_{a},
$$

and the monotoneity of $f_{5, c}$ follows from (1) and Lemma 5.1. By l'Hôpital's Rule, $f_{5, c}(0+)=\pi a / c$. Finally, since $2\left(1-a+a^{2}\right) \geq 3 / 2$, the result for $f_{5,3 / 2}$ follows.

When $a=\frac{1}{2}$, parts (1), (2), (3), (4), (6) of the next theorem are contained in [AVV, Theorems 3.21 (3), 5.13 (2), 3.30 (1), 5.16 (1), (2)], respectively.

Theorem 5.5. For each $a \in\left(0, \frac{1}{2}\right]$ the function

(1) $f_{1}(r) \equiv \mathcal{K}_{a}(r) / \sin (\pi a)+\log r^{\prime}$ is decreasing from $(0,1)$ onto $(R(a) / 2$, $\pi /(2 \sin (\pi a)))$.

(2) $f_{2}(r) \equiv \mu_{a}(r)+\log r$ is decreasing and concave from $(0,1)$ onto $(0, R(a) / 2)$.

(3) $f_{3}(r) \equiv m_{a}(r)+\log r$ is decreasing and concave from $(0,1)$ onto $(0, R(a) / 2)$.

(4) $f_{4}(r) \equiv \mu_{a}(r) / \log (1 / r)$ is increasing from $(0,1)$ onto $(1, \infty)$.

(5) $f_{5}(r) \equiv \mu_{a}(r)$ arth $r$ is increasing from $(0,1)$ onto $\left(0, \pi^{2} /\left(4 \sin ^{2}(\pi a)\right)\right)$.

(6) $f_{6}(r) \equiv \mu_{a}(r) / \log \left(e^{(R(a) / 2)} / r\right)$ is decreasing from $(0,1)$ onto $(0,1)$.

(7) $f_{7}(r)=\mathcal{K}_{a}(r)\left(\mu_{a}(r)+\log r\right)$ is increasing from $(0,1)$ onto $(\pi R(a) / 4$, $\left.\pi^{2} /(4 \sin (\pi a))\right)$.

(8) $f_{8}(r)=((\operatorname{arth} r) / r)\left(\mu_{a}(r)+\log r\right)$ is increasing from $(0,1)$ onto $\left(R(a) / 2, \pi^{2} /\left(4 \sin ^{2}(\pi a)\right)\right)$.

(9) $f_{9}(r) \equiv\left[(R(a) / 2)-\left(\mu_{a}(r)+\log r\right)\right] / r^{2}$ is increasing from $(0,1)$ onto $\left(\left(1-2 a+2 a^{2}\right) / 2, R(a) / 2\right)$. 
Proof. Part (1) follows from the fact that $B(a, b) F(a, b ; a+b ; r)+\log (1-r)$ is decreasing from $(0,1)$ onto $(R(a, b), B(a, b))$ [ABRVV, Theorem $1.3(2)]$. Parts (2), (3), and (6) were obtained in [QVu2, Corollary 3.12], [QVu3, Theorem 2.29 (1)], and [QVu3, Theorem 1.13 (3)], respectively, while parts (4) and (5) are implied by [QVu3, Theorem 1.13 (1)] and [QVu2, Theorem $1.28(2)]$, respectively.

For $(7)$, we write $f_{7}(r)=g_{7}(r) / h_{7}(r)$, where $g_{7}(r)=\mu_{a}(r)+\log r$ and $h_{7}(r)=1 / \mathcal{K}_{a}(r)$. Then $g_{7}(1-)=h_{7}(1-)=0$ and, after simplification,

$$
\frac{g_{7}^{\prime}(r)}{h_{7}^{\prime}(r)}=\frac{\left(\frac{\pi}{2}\right)^{2}-\left(r^{\prime} \mathcal{K}_{a}\right)^{2}}{2(1-a)\left(\mathcal{E}_{a}-r^{\prime 2} \mathcal{K}_{a}\right)}
$$

which is increasing by Lemma $5.2(7)$. Hence, by Lemma $5.1, f_{7}(r)$ is also increasing. Finally, $f_{7}(0+)=\pi R(a) / 4$ follows from (2) and (1.9), while $f_{7}(1-)=\pi^{2} /(4 \sin (\pi a))$, since

$$
\mathcal{K}_{a}(r) \log r=\left(r^{\prime 2} \mathcal{K}_{a}(r)\right) \frac{\log r}{r^{\prime 2}}
$$

tends to 0 as $r$ tends to 1, by Lemma 5.4 (1) and l'Hôpital's Rule.

Part (8) follows from (7) and Lemma $5.4(4)$.

For $(9), f_{9}(r)=g_{9}(r) / h_{9}(r)$, where $g_{9}(r)=(R(a) / 2)-\left(\mu_{a}(r)+\log r\right)$ and $h_{9}(r)=r^{2}$. Then $g_{9}(0)=h_{9}(0)=0$, and

$$
\frac{g_{9}^{\prime}(r)}{h_{9}^{\prime}(r)}=\frac{1}{2} \frac{1}{\left(r^{\prime} \mathcal{K}_{a}\right)^{2}} \frac{\left(\pi^{2} / 4\right)-\left(r^{\prime} \mathcal{K}_{a}\right)^{2}}{r^{2}}
$$

which is increasing, with $f_{9}(0+)=\pi^{2}\left(1-2 a+2 a^{2}\right) / 4$, by Lemma $5.2(14)$. The value $f_{9}(1-)=R(a) / 2$ is clear. Hence, the result follows from Lemma 5.1 .

Theorem 5.6. For each $a \in\left(0, \frac{1}{2}\right)$, the function $f(x) \equiv \mu_{a}(1 / \operatorname{ch} x)$ is increasing and concave from $(0, \infty)$ onto $(0, \infty)$. In particular, for $r, s \in$ $(0,1)$,

$$
\mu_{a}\left(\frac{r s}{1+r^{\prime} s^{\prime}}\right) \leq \mu_{a}(r)+\mu_{a}(s) \leq 2 \mu_{a}\left(\frac{\sqrt{2 r s}}{\sqrt{1+r s+r^{\prime} s^{\prime}}}\right)
$$

with equality in the second inequality if and only if $r=s$.

Proof. Let $r=1 / \operatorname{ch} x$ and $s=1 / \operatorname{ch} y$. Then

$$
f^{\prime}(x)=\frac{\pi^{2}}{4 r^{\prime} \mathcal{K}_{a}(r)^{2}},
$$

which is positive and increasing in $r$ by Lemma 5.4 (1), hence decreasing in $x$. Therefore, $f$ is increasing and concave on $(0, \infty)$. In particular, we have 
$f((x+y) / 2) \geq(f(x)+f(y)) / 2$, with equality if and only if $x=y$. Now

$$
\operatorname{ch}^{2}\left(\frac{x+y}{2}\right)=\frac{1+r s+r^{\prime} s^{\prime}}{2 r s} .
$$

Hence

$$
\frac{1}{2}(f(x)+f(y)) \leq f\left(\frac{x+y}{2}\right)
$$

gives

$$
\mu_{a}(r)+\mu_{a}(s) \leq 2 \mu_{a}\left(\sqrt{\frac{2 r s}{1+r s+r^{\prime} s^{\prime}}}\right),
$$

with equality if and only if $r=s$. Finally, since $f(0+)=0$ and since $f^{\prime}(x)$ is decreasing in $x$, it follows from Lemma 5.1 that $f(x) / x$ is decreasing on $(0, \infty)$. Hence $f(x+y) \leq f(x)+f(y)$ by $[\mathbf{A V V}$, Lemma 1.24].

Corollary 5.7. For each $a \in\left(0, \frac{1}{2}\right]$ and $r, s \in(0,1), \mu_{a}(r)+\mu_{a}(s) \leq$ $2 \mu_{a}(\sqrt{r s})$, with equality if and only if $r=s$.

Proof. Since $\mu_{a}$ is decreasing,

$$
\begin{gathered}
\mu_{a}\left(\sqrt{\frac{2 r s}{1+r s+r^{\prime} s^{\prime}}}\right) \leq \mu_{a}(\sqrt{r s}) \Longleftrightarrow 1+r s+r^{\prime} s^{\prime} \leq 2 \Longleftrightarrow r^{\prime} s^{\prime} \leq 1-r s \\
\Longleftrightarrow 1-r^{2}-s^{2}+r^{2} s^{2} \leq 1-2 r s+r^{2} s^{2} \Longleftrightarrow(r-s)^{2} \geq 0,
\end{gathered}
$$

with equality at each step if and only if $r=s$. Hence the result follows from Theorem 5.6.

\section{Modular functions.}

The next lemma gives some basic properties of the functions $\varphi_{K}^{a}(r)$ and $\eta_{K}^{a}(r)$, generalizing Theorem 10.5 (1) and Exercise 10.65 (13) in [AVV].

Lemma 6.1. For each $a \in(0,1 / 2], K \in(0, \infty), r \in(0,1), x \in(0, \infty)$, we have

$$
\begin{gathered}
\varphi_{K}^{a}(r)^{2}+\varphi_{1 / K}^{a}\left(r^{\prime}\right)^{2}=1, \\
\eta_{K}^{a}(x) \eta_{1 / K}^{a}(1 / x)=1 .
\end{gathered}
$$

Proof. Let $s=\varphi_{K}^{a}(r), u=\varphi_{1 / K}^{a}\left(r^{\prime}\right)$. Then $\mu_{a}(s)=\mu_{a}(r) / K$ and $\mu_{a}(u)=$ $K \mu_{a}\left(r^{\prime}\right)$. Hence, by (4.8),

$$
\mu_{a}(s) \mu_{a}(u)=\mu_{a}(r) \mu_{a}\left(r^{\prime}\right)=\frac{\pi^{2}}{4 \sin ^{2}(\pi a)}=\mu_{a}(s) \mu_{a}\left(s^{\prime}\right) .
$$

Thus $s^{\prime}=u$, so that (1) follows.

(2) Let $r=\sqrt{x /(x+1)}$. Then, by $(1), \eta_{K}^{a}(x)=\left(s / s^{\prime}\right)^{2}$ and $\eta_{1 / K}^{a}(1 / x)=$ $\left(s^{\prime} / s\right)^{2}$, so that $(2)$ follows. 
The next lemma will be needed for the proofs of some of the main results stated in the Introduction. Parts (1), (2), (3) generalize Lemma 10.7 (3), (1), (2), respectively, in [AVV].

Lemma 6.2. For each $a \in\left(0, \frac{1}{2}\right], K \in(1, \infty), r \in(0,1)$, let $s=\varphi_{K}^{a}(r)$ and $t=\varphi_{1 / K}^{a}(r)$. Then the function

(1) $f(r) \equiv \mathcal{K}_{a}(s) / \mathcal{K}_{a}(r)$ is increasing from $(0,1)$ onto $(1, K)$,

(2) $g(r) \equiv s^{\prime} \mathcal{K}_{a}(s)^{2} /\left(r^{\prime} \mathcal{K}_{a}(r)^{2}\right)$ is decreasing from $(0,1)$ onto $(0,1)$,

(3) $h(r) \equiv s \mathcal{K}_{a}^{\prime}(s)^{2} /\left(r \mathcal{K}_{a}^{\prime}(r)^{2}\right)$ is decreasing from $(0,1)$ onto $(1, \infty)$,

(4) $F(r) \equiv \mathcal{K}_{a}(t) / \mathcal{K}_{a}(r)$ is decreasing from $(0,1)$ onto $(1 / K, 1)$,

(5) $G(r) \equiv t^{\prime} \mathcal{K}_{a}(t)^{2} /\left(r^{\prime} \mathcal{K}_{a}(r)^{2}\right)$ is increasing from $(0,1)$ onto $(1, \infty)$,

(6) $H(r) \equiv t \mathcal{K}^{\prime}{ }_{a}(t)^{2} /\left(r \mathcal{K}^{\prime}{ }_{a}(r)^{2}\right)$ is increasing from $(0,1)$ onto $(0,1)$.

Proof. By Theorem 4.1 (1), (7), we have

$$
\begin{aligned}
& \mathcal{K}_{a}(r)^{2} f^{\prime}(r) \\
& =2(1-a)\left[\mathcal{K}_{a}(r) \frac{\mathcal{E}_{a}(s)-s^{\prime 2} \mathcal{K}_{a}(s)}{s s^{\prime 2}} \frac{d s}{d r}-\mathcal{K}_{a}(s) \frac{\mathcal{E}_{a}(r)-r^{\prime 2} \mathcal{K}_{a}(r)}{r r^{\prime 2}}\right] \\
& =\frac{2(1-a) \mathcal{K}_{a}(s)}{r r^{\prime 2} \mathcal{K}_{a}^{\prime}(r)}\left[\mathcal{K}_{a}^{\prime}(s)\left(\mathcal{E}_{a}(s)-s^{\prime 2} \mathcal{K}_{a}(s)\right)\right. \\
& \left.\quad-\mathcal{K}_{a}^{\prime}(r)\left(\mathcal{E}_{a}(r)-r^{\prime 2} \mathcal{K}_{a}(r)\right)\right] .
\end{aligned}
$$

Thus $f^{\prime}(r)$ is positive since $s>r$ and

$$
\mathcal{K}_{a}^{\prime}(x)\left(\mathcal{E}_{a}(x)-x^{\prime 2} \mathcal{K}_{a}(x)\right)=\left(x^{2} \mathcal{K}_{a}^{\prime}(x)\right) \frac{\mathcal{E}_{a}(x)-x^{\prime 2} \mathcal{K}_{a}(x)}{x^{2}}
$$

is increasing in $x$ by Lemmas 5.4 (1) and $5.2(1)$. Hence $f$ is increasing. The limiting values are clear.

Next, $g^{\prime}(r)$ is negative if and only if

$$
\begin{aligned}
\mathcal{K}_{a}^{\prime}(s)\left[s^{2} \mathcal{K}_{a}(s)-4(1-a)\right. & \left.\left(\mathcal{E}_{a}(s)-s^{\prime 2} \mathcal{K}_{a}(s)\right)\right] \\
& -\mathcal{K}_{a}^{\prime}(r)\left[r^{2} \mathcal{K}_{a}(r)-4(1-a)\left(\mathcal{E}_{a}(r)-r^{\prime 2} \mathcal{K}_{a}(r)\right)\right]
\end{aligned}
$$

is positive. This is true if

$$
g_{1}(r) \equiv \mathcal{K}_{a}^{\prime}(r)\left[r^{2} \mathcal{K}_{a}(r)-4(1-a)\left(\mathcal{E}_{a}(r)-r^{\prime 2} \mathcal{K}_{a}(r)\right)\right]
$$

is increasing. Now,

$$
g_{1}(r)=r^{2} \mathcal{K}_{a}(r) \mathcal{K}_{a}^{\prime}(r)\left[1-4(1-a) \frac{\mathcal{E}_{a}(r)-r^{\prime 2} \mathcal{K}_{a}(r)}{r^{2} \mathcal{K}_{a}(r)}\right],
$$

which is positive and increasing, by Lemmas $5.2(4)$ and $5.4(1)$.

(3) Since $h(r)=1 / g\left(s^{\prime}\right), F(r)=1 / f(t), G(r)=1 / g(t)$, and $H(r)=$ $1 / h(t)$, parts (3)-(6) follow from parts (1) and (2). 
6.3. Proof of Theorem 1.14. Let $r=e^{-x}$ and $s=\varphi_{K}^{a}(r)$. Then

$$
f^{\prime}(x)=\frac{1}{K}\left(\frac{s^{\prime} \mathcal{K}_{a}(s)}{r^{\prime} \mathcal{K}_{a}(r)}\right)^{2},
$$

which is positive and increasing in $x$, by Lemma $6.2(1),(2)$. Hence $f$ is strictly increasing and convex. Thus $f((x+y) / 2) \leq(f(x)+f(y)) / 2$, so that

$$
1 / \varphi_{K}^{a}\left(e^{-(x+y) / 2}\right) \leq 1 /\left(\varphi_{K}^{a}\left(e^{-x}\right) \varphi_{K}^{a}\left(e^{-y}\right)\right)^{1 / 2} .
$$

Now putting $r=e^{-x}$ and $t=e^{-y}$ gives the result. The proof for $g(x)$ is similar.

6.4. Proof of Theorem 1.15. Let $t=r x, u=\varphi_{K}^{a}(t)$, and $s=\varphi_{K}^{a}(x)$. Then

$$
\begin{aligned}
\frac{f^{\prime}(x)}{f(x)} & =\frac{1}{u} \frac{1}{K} \frac{r u u^{\prime 2} \mathcal{K}_{a}(u)^{2}}{t t^{\prime 2} \mathcal{K}_{a}(t)^{2}}-\frac{1}{s} \frac{1}{K} \frac{s s^{\prime 2} \mathcal{K}_{a}(s)^{2}}{x x^{\prime 2} \mathcal{K}_{a}(x)^{2}} \\
& =\frac{1}{K x}\left[\left(\frac{u^{\prime} \mathcal{K}_{a}(u)}{t^{\prime} \mathcal{K}_{a}(t)}\right)^{2}-\left(\frac{s^{\prime} \mathcal{K}_{a}(s)}{x^{\prime} \mathcal{K}_{a}(x)}\right)^{2}\right],
\end{aligned}
$$

which is positive by Lemma $6.2(1),(2)$ since $t<x$. The proof for $g(x)$ is similar.

The following inequalities generalize identities satisfied by the functions $\mu$ and $\varphi_{K}(r)$ (cf. [LV, pp. 60, 61], [AVV, Theorem 10.5 (3), (7)]).

Theorem 6.5. For $a \in\left(0, \frac{1}{2}\right], r \in(0,1), K \in(0, \infty)$,

$$
\begin{aligned}
\frac{1}{2} \mu_{a}(r) & \leq \mu_{a}\left(\frac{2 \sqrt{r}}{1+r}\right) \leq \mu_{a}(r) . \\
\mu_{a}\left(r^{\prime}\right) & \leq \mu_{a}\left(\frac{1-r}{1+r}\right) \leq 2 \mu_{a}\left(r^{\prime}\right) . \\
\varphi_{K}^{a}(r) & \leq \varphi_{K}^{a}\left(\frac{2 \sqrt{r}}{1+r}\right) \leq \varphi_{2 K}^{a}(r) . \\
\varphi_{K / 2}\left(r^{\prime}\right) & \leq \varphi_{K}^{a}\left(\frac{1-r}{1+r}\right) \leq \varphi_{K}^{a}\left(r^{\prime}\right) .
\end{aligned}
$$

Proof. Inequalities (1) and (2) were obtained in [QVu2, Theorem 1.14 (1)], and (3), (4) follow from (1), (2), respectively if we divide by $K$ and apply $\mu_{a}^{-1}$.

Theorem 6.6. For each $a \in\left(0, \frac{1}{2}\right], r \in(0,1)$, let $f:(0, \infty) \rightarrow \mathbf{R}$ be defined by

$$
f(K) \equiv \frac{K \log r-\log \varphi_{1 / K}^{a}(r)}{K-1} .
$$


Then $f$ is increasing, with $f(0+)=0$,

$$
\lim _{K \rightarrow 1} f(K)=\log r+m_{a}(r), \text { and } \lim _{K \rightarrow \infty} f(K)=\log r+\mu_{a}(r) .
$$

In particular, for $K \in(1, \infty), r \in(0,1), a \in\left(0, \frac{1}{2}\right]$,

(1) $1<e^{(K-1)\left(m_{a}(r)+\log r\right)}<\frac{r^{K}}{\varphi_{1 / K}^{a}(r)}<e^{(K-1)\left(\mu_{a}(r)+\log r\right)}<e^{(K-1) R(a) / 2}$

and

$$
1<\frac{\varphi_{K}^{a}(r)}{r^{1 / K}}<e^{(1-1 / K)\left(\log r+m_{a}(r)\right)}<e^{(1-1 / K) R(a) / 2} .
$$

Proof. Since $\mu_{a}^{-1}(0+)=1$, it is clear that $f(0+)=0$. Now let $t=\varphi_{1 / K}^{a}(r)$, and write $f(K)=g(K) / h(K)$, where $g(K)=K \log r-\log \varphi_{1 / K}^{a}(r)$ and $h(K)=K-1$. Then $g(1)=h(1)=0$, and

$$
\frac{g^{\prime}(K)}{h^{\prime}(K)}=\log r+\frac{4}{\pi^{2}}\left(t^{\prime} \mathcal{K}_{a}(t)\right)^{2} \mu_{a}(r),
$$

which is increasing in $K$ on $(0, \infty)$ by Lemma $5.4(1)$ and the fact that $t$ is decreasing in $K$. Hence $f$ is increasing, by Lemma 5.1 .

Next, by l'Hôpital's Rule,

$$
\lim _{K \rightarrow 1} f(K)=\log r+\frac{2}{\pi \sin (\pi a)} r^{\prime 2} \mathcal{K}_{a} \mathcal{K}_{a}^{\prime}=\log r+m_{a}(r),
$$

while

$$
\lim _{K \rightarrow \infty} f(K)=\log r+\frac{4}{\pi^{2}} \mathcal{K}_{a}(0)^{2} \mu_{a}(r)=\log r+\mu_{a}(r) .
$$

Inequalities (1), (2) follow from the above argument and Theorems 5.5 (3), (2).

The next theorem is a generalization of a result due to Hübner and He (cf. [AVV, Theorem 10.9 (1)]).

Theorem 6.7. For each $a \in\left(0, \frac{1}{2}\right]$ and $K \in(1, \infty)$, let $f, g$ be defined on $(0,1]$ by

$$
f(r)=r^{-1 / K} \varphi_{K}^{a}(r) \quad \text { and } \quad g(r)=r^{-K} \varphi_{1 / K}^{a}(r) .
$$

Then $f$ is decreasing and $g$ is increasing, with $f((0,1])=\left[1, e^{(1-1 / K) R(a) / 2}\right)$ and $g((0,1])=\left(e^{(1-K) R(a) / 2}, 1\right]$.

Proof. First, let $s=\varphi_{K}^{a}(r)$. Then

$$
\frac{f^{\prime}(r)}{f(r)}=\frac{1}{K r}\left(\left(\frac{s^{\prime} \mathcal{K}_{a}(s)}{r^{\prime} \mathcal{K}_{a}(r)}\right)^{2}-1\right),
$$


which is negative by Lemma $6.2(1),(2)$. Clearly $f(1)=1$, while

$$
\begin{aligned}
\lim _{r \rightarrow 0} \log \left(r^{-1 / K} s\right) & =\lim _{r \rightarrow 0}\left[\left(\mu_{a}(s)+\log s\right)-\frac{1}{K}\left(\mu_{a}(r)+\log r\right)\right] \\
& =\frac{R(a)}{2}\left(1-\frac{1}{K}\right)
\end{aligned}
$$

by (1.5) and Theorem 5.5 (2).

Next, let $t=\varphi_{1 / K}^{a}(r)$. Then $r=\varphi_{K}^{a}(t)$. Hence $g(r)=\left(t^{-1 / K} \varphi_{K}^{a}(t)\right)^{-K}$, so that the assertion about $g(r)$ follows from the properties of $f(r)$ already proved.

Theorem 6.8. For each $a \in\left(0, \frac{1}{2}\right]$ and $K \in(1, \infty)$, the function $f(r) \equiv$ $\varphi_{K}^{a}(r)$ is increasing and concave from $(0,1)$ onto $(0,1)$, and $g(r) \equiv \varphi_{1 / K}^{a}(r)$ is increasing and convex from $(0,1)$ onto $(0,1)$.

Proof. By Theorem $4.1(7), f^{\prime}(r)=(1 / K) s s^{\prime 2} \mathcal{K}_{a}(s)^{2} /\left(r r^{\prime 2} \mathcal{K}_{a}(r)^{2}\right)$, which is positive and decreasing by Theorem 6.6 and Lemma $6.2(1),(2)$. The proof for $g(r)$ is similar.

Corollary 6.9. For each $a \in\left(0, \frac{1}{2}\right], K \in(1, \infty)$,

$$
\begin{aligned}
& \lim _{r \rightarrow 0+} \frac{\partial \varphi_{K}^{a}(r)}{\partial r}=\infty, \quad \lim _{r \rightarrow 0+} \frac{\partial \varphi_{1 / K}^{a}(r)}{\partial r}=0, \\
& \lim _{r \rightarrow 1-} \frac{\partial \varphi_{K}^{a}(r)}{\partial r}=0, \quad \lim _{r \rightarrow 1-} \frac{\partial \varphi_{1 / K}^{a}(r)}{\partial r}=\infty .
\end{aligned}
$$

Proof. Let $s=\varphi_{K}^{a}(r)$. From Theorems $4.1(7)$ and 6.7 we have

$$
\lim _{r \rightarrow 0+} \frac{\partial \varphi_{K}^{a}(r)}{\partial r}=\frac{1}{K} \lim _{r \rightarrow 0+} \frac{s}{r^{1 / K}} \lim _{r \rightarrow 0+} r^{-1+1 / K}=\infty
$$

and

$$
\lim _{r \rightarrow 1-} \frac{\partial \varphi_{K}^{a}(r)}{\partial r}=K \lim _{r \rightarrow 1-}\left(\frac{s^{\prime}}{r^{\prime K}}\right)^{2} \lim _{r \rightarrow 1-} r^{\prime 2(K-1)}=0 .
$$

The proof for $\varphi_{1 / K}^{a}(r)$ is similar.

6.10. Proof of Theorem 1.16. Let $y=g(x)=p\left(\varphi_{K}^{a}(q(x))\right)=p(s)$, where $s=\varphi_{K}^{a}(r), r=q(x)$. Then $q(y)=s$, so that $q^{\prime}(y) d y / d x=d s / d x=$ $(d s / d r)(d r / d x)$. Thus

$$
q^{\prime}(y) g^{\prime}(x)=\frac{1}{K} \frac{s s^{\prime 2} \mathcal{K}_{a}(s)^{2}}{r r^{\prime 2} \mathcal{K}_{a}(r)^{2}} \frac{d r}{d x}
$$

by Theorem 4.1 (7). Now

$$
r^{2}=q(x)^{2}=\frac{e^{x}}{e^{x}+1}=1-\frac{1}{e^{x}+1}
$$


giving

$$
2 r \frac{d r}{d x}=r^{2} r^{\prime 2}
$$

Thus $q^{\prime}(x)=r r^{2} / 2$ and $q^{\prime}(y)=s s^{2} / 2$. Hence, we get

$$
g^{\prime}(x)=\frac{1}{K} \frac{\mathcal{K}_{a}(s)^{2}}{\mathcal{K}_{a}(r)^{2}}
$$

which increases from $1 / K$ to $K$, by Lemma $6.2(1)$. The proof for $h(x)$ is similar.

Remark 6.11. Let $p, q, g, h$ be the functions defined in Theorem 1.16, and let $s=\varphi_{K}^{a}(1 / \sqrt{2})$. Then, by Theorem 1.16 and integration, we obtain

$$
\begin{aligned}
& \frac{x}{K}+2 \log \frac{s}{s^{\prime}} \leq g(x) \leq K x+2 \log \frac{s}{s^{\prime}}, \\
& \frac{x}{K}-2 \log \frac{s}{s^{\prime}} \leq h(x) \leq K x-2 \log \frac{s}{s^{\prime}} .
\end{aligned}
$$

In the next result we obtain further bounds for the functions $g$ and $h$.

Theorem 6.12. Let $p:(0,1) \rightarrow(-\infty, \infty)$ and $q:(-\infty, \infty) \rightarrow(0,1)$ be given by $p(x)=2 \log \left(x / x^{\prime}\right)$ and $q(x)=p^{-1}(x)=\sqrt{e^{x} /\left(e^{x}+1\right)}$, respectively, and for $a \in\left(0, \frac{1}{2}\right], K \in(1, \infty)$, let $g, h:(-\infty, \infty) \rightarrow(-\infty, \infty)$ be defined by $g(x)=p\left(\varphi_{K}^{a}(q(x))\right)$ and $h(x)=p\left(\varphi_{1 / K}^{a}(q(x))\right)$. Then

$$
g(x) \geq\left\{\begin{array}{ll}
K x, & x \geq 0, \\
\frac{x}{K}, & x<0,
\end{array} \quad \text { and } \quad h(x) \leq \begin{cases}\frac{x}{K}, & x \geq 0 \\
K x, & x<0\end{cases}\right.
$$

Proof. We only give the proof for $g$, since the proof for $h$ is similar. First, if $x>0$, then

$$
\begin{aligned}
g(x) \geq K x & \Leftrightarrow \varphi_{K}^{a}(q(x)) \geq q(K x) \\
& \Leftrightarrow \mu_{a}^{-1}\left(\frac{1}{K} \mu_{a}\left(\sqrt{\frac{e^{x}}{e^{x}+1}}\right)\right) \geq \sqrt{\frac{e^{K x}}{e^{K x}+1}} \\
& \Leftrightarrow \mu_{a}\left(\sqrt{\frac{e^{x}}{e^{x}+1}}\right) \leq K \mu_{a}\left(\sqrt{\frac{e^{K x}}{e^{K x}+1}}\right) .
\end{aligned}
$$

This will be true if $f(K) \equiv K \mu_{a}\left(\sqrt{e^{K x} /\left(e^{K x}+1\right)}\right)$ is increasing on $[1, \infty)$.

Now, with $r=\sqrt{e^{K x} /\left(e^{K x}+1\right)}, r^{2}=e^{K x} /\left(e^{K x}+1\right), r^{\prime 2}=1 /\left(e^{K x}+1\right)$, we have

$$
2 r \frac{d r}{d K}=\frac{x e^{K x}}{\left(e^{K x}+1\right)^{2}}=x r^{2} r^{\prime 2}, \quad \frac{d r}{d K}=\frac{x r r^{\prime 2}}{2} .
$$




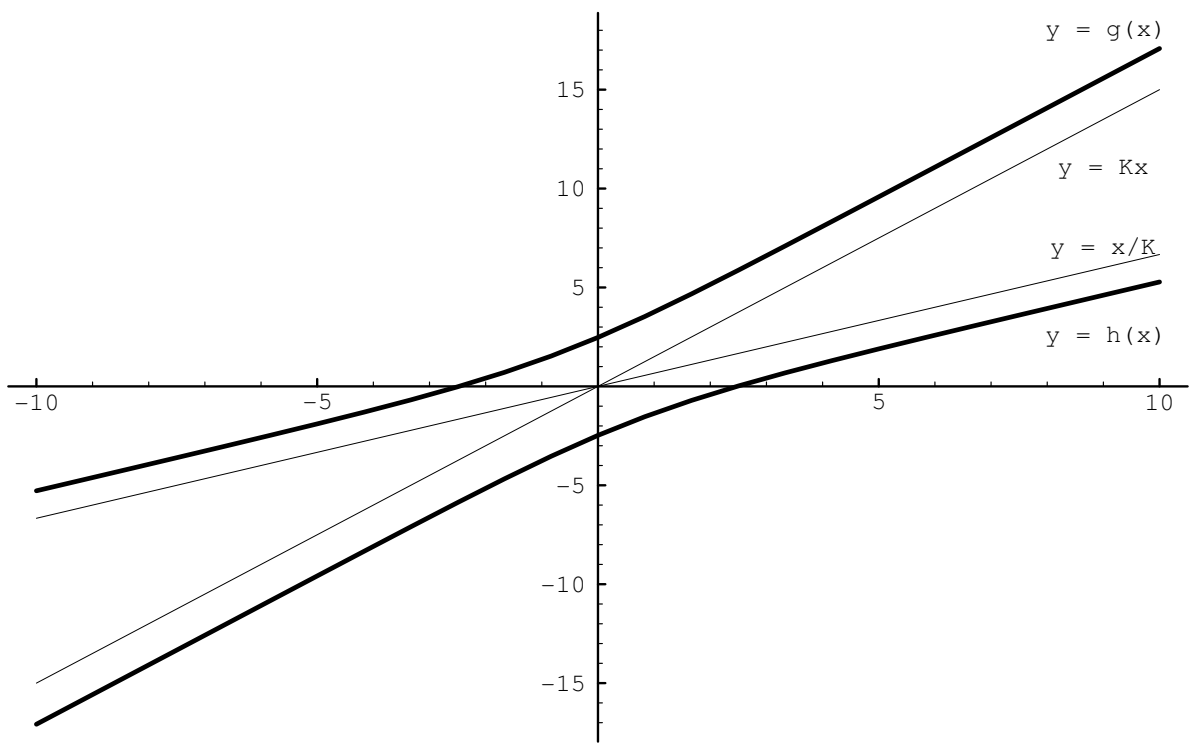

Figure 1. The graphs of the functions $g, h$, and those of the lines $K x, x / K$, in Theorem 6.12 , where $a=0.25$ and $K=1.5$.

Hence

$$
\begin{aligned}
f^{\prime}(K) & =\mu_{a}(r)-\frac{K \pi^{2}}{4 r r^{\prime 2} \mathcal{K}_{a}(r)^{2}} \frac{d r}{d K} \\
& =\mu_{a}(r)-\frac{\pi^{2} K x}{8 \mathcal{K}_{a}(r)^{2}}=\frac{\pi}{2}\left[\frac{\mathcal{K}_{a}^{\prime}(r)}{\mathcal{K}_{a}(r) \sin (\pi a)}-\frac{\pi K x}{4 \mathcal{K}_{a}(r)^{2}}\right],
\end{aligned}
$$

and

$$
\begin{aligned}
& \frac{\pi}{2 \mathcal{K}_{a}(r)^{2} \sin (\pi a)}\left[\mathcal{K}_{a}(r) \mathcal{K}_{a}^{\prime}(r)-\frac{\pi}{4} K x \sin (\pi a)\right]>0 \\
& \Leftrightarrow \mathcal{K}_{a}(r) \mathcal{K}_{a}^{\prime}(r)>\frac{\pi}{4}(K x) \sin (\pi a) \\
& \Leftrightarrow \mathcal{K}_{a}(r) \mathcal{K}_{a}^{\prime}(r)>\frac{\pi}{2}\left(\log \frac{r}{r^{\prime}}\right) \sin (\pi a),
\end{aligned}
$$

for $r \in(1 / \sqrt{2}, 1)$. Now from (4.9) and Theorem 5.5 (3),

$$
\begin{aligned}
\mathcal{K}_{a}(r) \mathcal{K}_{a}^{\prime}(r) & =\frac{\pi}{2}\left(m_{a}(r)+m_{a}\left(r^{\prime}\right)\right) \sin (\pi a) \\
& >\frac{\pi}{2}\left(\log \frac{1}{r r^{\prime}}\right) \sin (\pi a)>\frac{\pi}{2}\left(\log \frac{r}{r^{\prime}}\right) \sin (\pi a),
\end{aligned}
$$

which proves the first inequality for $g(x)$. 
Next, $g(-x) \geq-x / K, x>0$

$$
\begin{aligned}
& \Leftrightarrow \varphi_{K}^{a}\left(\sqrt{\frac{e^{-x}}{e^{-x}+1}}\right) \geq \sqrt{\frac{e^{-x / K}}{e^{-x / K}+1}}, x>0 \\
& \Leftrightarrow \mu_{a}^{-1}\left(\frac{1}{K} \mu_{a}\left(\sqrt{\frac{1}{e^{x}+1}}\right)\right) \geq \sqrt{\frac{1}{e^{x / K}+1}} \\
& \Leftrightarrow \mu_{a}\left(\frac{1}{\sqrt{e^{x}+1}}\right) \leq K \mu_{a}\left(\sqrt{\frac{1}{e^{x / K}+1}}\right) .
\end{aligned}
$$

This will be true if $F(K) \equiv K \mu_{a}\left(1 / \sqrt{e^{x / K}+1}\right)$ is increasing on $[1, \infty)$. Let $1 / \sqrt{e^{x / K}+1}=t$, so that $t \in(0,1 / \sqrt{2})$. Now $t^{2}=1 /\left(e^{x / K}+1\right), t^{\prime 2}=$ $e^{x / K} /\left(e^{x / K}+1\right)$, and

$$
\begin{aligned}
F^{\prime}(K) & =\mu_{a}(t)-\frac{K \pi^{2}}{4 t t^{\prime 2} \mathcal{K}_{a}(t)} \frac{d t}{d K} \\
& =\frac{\pi}{2 \sin (\pi a)} \frac{\mathcal{K}_{a}^{\prime}(t)}{\mathcal{K}_{a}(t)}-\frac{K \pi^{2}}{4 t t^{\prime 2} \mathcal{K}_{a}(t)^{2}} \frac{d t}{d K} .
\end{aligned}
$$

Now

$$
2 t \frac{d t}{d K}=\frac{x}{K^{2}} \frac{e^{x / K}}{\left(e^{x / K}+1\right)^{2}}=\frac{x}{K^{2}} t^{2} t^{\prime 2}, \frac{d t}{d K}=\frac{x}{K^{2}} \frac{t t^{\prime 2}}{2} .
$$

Hence

$$
\begin{aligned}
F^{\prime}(K) & =\frac{\pi}{2 \sin (\pi a)} \frac{\mathcal{K}_{a}^{\prime}(t)}{\mathcal{K}_{a}(t)}-\frac{\pi^{2} x}{8 K \mathcal{K}_{a}(t)^{2}} \\
& =\frac{\pi}{2(\sin (\pi a)) \mathcal{K}_{a}(t)^{2}}\left[\mathcal{K}_{a}(t) \mathcal{K}_{a}^{\prime}(t)-\frac{\pi(\sin (\pi a)) x}{4 K}\right] \\
& =\frac{\pi}{2(\sin (\pi a)) \mathcal{K}_{a}(t)^{2}}\left[\mathcal{K}_{a}(t) \mathcal{K}_{a}^{\prime}(t)-\frac{\pi}{2}\left(\log \frac{t^{\prime}}{t}\right) \sin (\pi a)\right]
\end{aligned}
$$

Hence $F^{\prime}(K)>0$ if and only if $\mathcal{K}_{a}(t) \mathcal{K}_{a}^{\prime}(t)>(\pi / 2)(\sin (\pi a)) \log \left(t^{\prime} / t\right)$. Now, by (4.9) and Theorem 5.5 (3), we have

$$
\begin{aligned}
\mathcal{K}_{a}(t) \mathcal{K}_{a}^{\prime}(t) & =\frac{\pi \sin (\pi a)}{2}\left(m_{a}(t)+m_{a}\left(t^{\prime}\right)\right) \\
& >\frac{\pi}{2}\left(\log \frac{1}{t t^{\prime}}\right) \sin (\pi a)>\frac{\pi}{2}\left(\log \frac{t^{\prime}}{t}\right) \sin (\pi a) .
\end{aligned}
$$


Theorem 6.13. For each $a \in\left(0, \frac{1}{2}\right], K \in(1, \infty)$, let $f$ and $g$ be defined on $(0,1)$ by

$$
f(r)=\frac{\arcsin \left(\varphi_{K}^{a}(r)\right)}{\arcsin \left(r^{1 / K}\right)}, g(r)=\frac{\arcsin \left(\varphi_{1 / K}^{a}(r)\right)}{\arcsin \left(r^{K}\right)} .
$$

Then $f$ is decreasing and $g$ is increasing, with $f((0,1])=\left[1, e^{(1-1 / K) R(a) / 2}\right)$ and $g((0,1])=\left(e^{(1-K) R(a) / 2}, 1\right]$.

Proof. To prove the monotoneity for $f$, let $s=\varphi_{K}^{a}(r)$ and $f(r)=$ $f_{1}(r) / f_{2}(r)$, where $f_{1}(r)=\arcsin (s)$ and $f_{2}(r)=\arcsin \left(r^{1 / K}\right)$. Then $f_{1}(0)=$ $f_{2}(0)=0$, and

$$
\frac{f_{1}^{\prime}(r)}{f_{2}^{\prime}(r)}=\frac{s}{r^{1 / K}}\left(\frac{1-r^{2 / K}}{1-r^{2}}\right)^{1 / 2} \frac{s^{\prime} \mathcal{K}_{a}(s)^{2}}{r^{\prime} \mathcal{K}_{a}(r)^{2}},
$$

which is decreasing, by Lemma 6.2 (2) and Theorem 6.7. The limiting values follow from l'Hôpital's Rule and Theorem 6.7. The proof for $g$ is similar.

Theorem 6.14. For each $a \in\left(0, \frac{1}{2}\right], K \in(1, \infty)$, let $f$ and $g$ be defined on $(0, \infty)$ by

$$
f(x)=\operatorname{arth} \varphi_{K}^{a}(\operatorname{th} x) \quad \text { and } \quad g(x)=\operatorname{arth} \varphi_{1 / K}^{a}(\operatorname{th} x) .
$$

Then $f$ and $g$ are increasing automorphisms; $f$ is concave and $g$ is convex. In particular,

$$
f(x+y) \leq f(x)+f(y) \leq 2 f\left(\frac{x+y}{2}\right)
$$

and

$$
g(x+y) \geq g(x)+g(y) \geq 2 g\left(\frac{x+y}{2}\right)
$$

for all $x, y \in(0, \infty)$.

Proof. For $f$, let $r=\operatorname{th} x$ and $s=\varphi_{K}^{a}(r)$. Then

$$
f^{\prime}(x)=\frac{1}{s^{\prime 2}} K \frac{s s^{2} \mathcal{K}_{a}^{\prime}(s)^{2}}{r r^{\prime 2} \mathcal{K}_{a}^{\prime}(r)^{2}} r^{\prime 2}=K \frac{s \mathcal{K}_{a}^{\prime}(s)^{2}}{r \mathcal{K}_{a}^{\prime}(r)^{2}},
$$

which is positive and decreasing, by Lemma 6.2 (3). Hence $f$ is increasing and concave, so $f(x) / x$ is decreasing by Lemma 5.1. The double inequality (6.15) follows from [AVV, Lemma 1.24] and the concavity of $f$. The proof for $g$ is similar.

Remark 6.17 (Cf. [AVV, Theorem 10.12]). The inequalities in Theorem 6.14 can be simplified to

$$
\varphi_{K}^{a}\left(\frac{r+s}{1+r s}\right) \leq \frac{\varphi_{K}^{a}(r)+\varphi_{K}^{a}(s)}{1+\varphi_{K}^{a}(r) \varphi_{K}^{a}(s)}
$$


and

$$
\frac{\varphi_{K}^{a}(r)+\varphi_{K}^{a}(s)}{1+\varphi_{K}^{a}(r) \varphi_{K}^{a}(s)+\varphi_{1 / K}^{a}\left(r^{\prime}\right) \varphi_{1 / K}^{a}\left(s^{\prime}\right)} \leq \varphi_{K}^{a}\left(\frac{r+s}{1+r s+r^{\prime} s^{\prime}}\right),
$$

for $a \in\left(0, \frac{1}{2}\right], r, s, \in(0,1)$, and $K \in(1, \infty)$. The inequalities are reversed if we replace $K$ by $1 / K$.

Theorem 6.18. For $a \in\left(0, \frac{1}{2}\right], r \in(0,1), K \in(1, \infty)$,

$$
\operatorname{th}(K \operatorname{arth} r)<\varphi_{K}^{a}(r) \text {. }
$$

The inequality is reversed if we replace $K$ by $1 / K$.

Proof. Let $s=\varphi_{K}^{a}(r)$. Then $s>r$, and it follows from (1.5) and Theorem 5.5 (5) that

$$
\frac{1}{K} \mu_{a}(r) \operatorname{arth} s=\mu_{a}(s) \operatorname{arth} s>\mu_{a}(r) \operatorname{arth} r,
$$

and the result follows if we solve for $s$.

The reverse inequality follows if we let $\varphi_{K}^{a}(r)=x$ in the inequality just proved and solve for $r$.

The next result generalizes Theorem 10.24 in [AVV].

Theorem 6.19. For each $a \in\left(0, \frac{1}{2}\right]$ and $K \in(1, \infty)$, the function

$$
f(x) \equiv \frac{\log \left(\eta_{K}^{a}(x) / \eta_{K}^{a}(1)\right)}{\log x}
$$

is increasing from $(0, \infty)$ onto $(1 / K, K)$, while

$$
g(x) \equiv \frac{\log \left(\eta_{1 / K}^{a}(x) / \eta_{1 / K}^{a}(1)\right)}{\log x}
$$

is decreasing from $(0, \infty)$ onto $(1 / K, K)$. In particular,

$$
\begin{gathered}
\eta_{K}^{a}(1) \min \left\{x^{K}, x^{1 / K}\right\} \leq \eta_{K}^{a}(x) \leq \eta_{K}^{a}(1) \max \left\{x^{K}, x^{1 / K}\right\}, \\
\eta_{1 / K}^{a}(1) \min \left\{x^{K}, x^{1 / K}\right\} \leq \eta_{1 / K}^{a}(x) \leq \eta_{1 / K}^{a}(1) \max \left\{x^{K}, x^{1 / K}\right\}
\end{gathered}
$$

for all $x \in(0, \infty), a \in\left(0, \frac{1}{2}\right], K \in[1, \infty)$.

Proof. Let $r=\sqrt{x /(x+1)}, s=\varphi_{K}^{a}(r)$, and $f(x)=G(x) / H(x)$, where $G(x)=\log \left(\eta_{K}^{a}(x) / \eta_{K}^{a}(1)\right)$ and $H(x)=\log x$. Then $G(1)=H(1)=0$ and

$$
\frac{G^{\prime}(x)}{H^{\prime}(x)}=\frac{1}{K}\left(\frac{\mathcal{K}_{a}(s)}{\mathcal{K}_{a}(r)}\right)^{2}
$$

which is increasing from $(0, \infty)$ onto $(1 / K, K)$ by Lemma $6.2(1)$. Hence $f$ is increasing by Lemma 5.1, while $f(0+)=1 / K$ and $\lim _{x \rightarrow \infty} f(x)=K$ follow from l'Hôpital's Rule. The proof for $g(x)$ is similar. 
Theorem 6.20. For $a \in\left(0, \frac{1}{2}\right], x \in(0, \infty), K \in[1, \infty)$, we have

$$
1 \leq \frac{\eta_{K}^{a}(x)}{x^{1 / K}(x+1)^{K-1 / K}} \leq e^{R(a)(K-1 / K)}
$$

where $R(a)$ is as in (3.4).

Proof. By (1.13) and Lemma 6.1 (1) we have $\eta_{K}^{a}(x)=\left(\varphi_{K}^{a}(r) / \varphi_{1 / K}^{a}\left(r^{\prime}\right)\right)^{2}$, where $r=\sqrt{x /(x+1)}$ and $r^{\prime}=1 / \sqrt{x+1}$. Next,

$$
r^{1 / K} \leq \varphi_{K}^{a}(r) \leq e^{(1-1 / K) R(a) / 2} r^{1 / K}
$$

and

$$
e^{(1-K) R(a) / 2} r^{\prime K} \leq \varphi_{1 / K}^{a}\left(r^{\prime}\right) \leq r^{\prime K}
$$

by Theorem 6.6. Hence the result follows when we divide and substitute for $r$ in terms of $x$.

Theorem 6.21. For each $a \in\left(0, \frac{1}{2}\right], K \in(1, \infty)$, let $f, g$ be defined on $(0,1)$ by

$$
f(r)=\varphi_{K}^{a}(r) \operatorname{ch}\left(\frac{1}{K} \operatorname{arch}\left(\frac{1}{r}\right)\right)
$$

and

$$
g(r)=\varphi_{1 / K}^{a}(r) \operatorname{ch}\left(K \operatorname{arch}\left(\frac{1}{r}\right)\right) .
$$

Then $f$ is decreasing and $g$ is increasing, with $f((0,1))=\left(1, e^{(1-1 / K) R(a) / 4}\right)$ and $g((0,1))=\left(e^{(1-K) R(a) / 4}, 1\right)$.

Proof. To prove the assertion for $f$, we first let $s=\varphi_{K}^{a}(r)$ and $1 / t=$ $\operatorname{ch}((1 / K)$ arch $(1 / r))$. Then $s^{\prime}=\varphi_{1 / K}^{a}\left(r^{\prime}\right)$ and $t^{\prime}=\operatorname{th}\left((1 / K)\right.$ arth $\left.\left(r^{\prime}\right)\right)$. Now, by differentiation and simplification,

$$
K \operatorname{trr} f^{\prime}(r)=s t^{\prime}\left[\frac{s^{\prime}}{t^{\prime}} \frac{s^{\prime} \mathcal{K}_{a}(s)^{2}}{r^{\prime} \mathcal{K}_{a}(r)^{2}}-1\right],
$$

which is negative, by Lemma $6.2(2)$ and Theorem 6.18. Hence $f$ is decreasing. The limiting values follow from the expression

$$
\operatorname{ch}\left(\frac{1}{K} \operatorname{arch}\left(\frac{1}{r}\right)\right)=\frac{\left(1+r^{\prime}\right)^{1 / K}+\left(1-r^{\prime}\right)^{1 / K}}{2 r^{1 / K}},
$$

and Theorem 6.7. The proof for $g$ is similar.

Theorem 6.22. Let $\mathcal{M}$ be a set with a multiplication operation and let $\left\{f_{K}: K \in \mathcal{M}\right\}$ be a collection of increasing functions $f_{K}:(0,1) \rightarrow(0,1)$, such that $f_{K} \circ f_{L}=f_{K L}$ and such that $f_{K}(x) / x$ is decreasing (respectively, increasing). Then

$$
f_{K}(x) f_{L}(y) \leq f_{K L}(x y),
$$

(respectively, $f_{K}(x) f_{L}(y) \geq f_{K L}(x y)$ ). 
Proof. Let $f_{K}(x) / x$ be decreasing for each $K \in \mathcal{M}$. Since $x y \leq x$, we have $f_{K}(x) / x \leq f_{K}(x y) /(x y)$. Thus $f_{K}(x) y \leq f_{K}(x y)$. Hence

$$
f_{K}(x) f_{L}(y) \leq f_{K}\left(x f_{L}(y)\right) \leq f_{K}\left(f_{L}(x y)\right)=f_{K L}(x y) .
$$

The other case is similar.

Corollary 6.23. For $a \in\left(0, \frac{1}{2}\right], x, y \in(0,1), K, L \in[1, \infty)$,

$$
\begin{gathered}
\varphi_{K}^{a}(x) \varphi_{L}^{a}(y) \leq \varphi_{K L}^{a}(x y), \\
\varphi_{1 / K}^{a}(x) \varphi_{1 / L}^{a}(y) \geq \varphi_{1 /(K L)}^{a}(x y) .
\end{gathered}
$$

\section{Dependence on $a$.}

In this section we study the generalized elliptic integrals as functions of the parameter $a$.

Lemma 7.1. For each nonnegative integer $n$, the function $f_{n}(x) \equiv$ $-(-x, n+1)(x, n+1)$ is positive and increasing on $\left[0, \frac{1}{2}\right]$. The function $g_{n}(x) \equiv(x, n+1)(1-x, n+1)$ is positive, increasing on $\left[0, \frac{1}{2}\right]$ and decreasing on $\left[\frac{1}{2}, 1\right]$. For $n \geq 1$, the function $f_{n}$ is positive and decreasing on $[1 / \sqrt{2}, 1]$. But $f_{0}$ is positive and increasing on $[0,1]$.

Proof. We have

$$
f_{n}(x)=x^{2}\left(1-x^{2}\right)\left(2^{2}-x^{2}\right) \cdots\left(n^{2}-x^{2}\right) .
$$

On $[1 / \sqrt{2}, 1], x^{2}\left(1-x^{2}\right)$ is decreasing, hence so is $f_{n}$ if $n \geq 1$. Next, on $\left(0, \frac{1}{2}\right)$,

$$
\frac{f_{n}^{\prime}(x)}{2 f_{n}(x)}=\frac{1}{x}-\sum_{k=1}^{n} \frac{x}{k^{2}-x^{2}},
$$

which is clearly decreasing. Hence

$$
\frac{f_{n}^{\prime}(x)}{2 f_{n}(x)}>2-2 \sum_{k=1}^{n} \frac{1}{4 k^{2}-1}=2-\left(1-\frac{1}{2 n+1}\right)>0 .
$$

Clearly, $f_{0}(x)=x^{2}$, which is increasing on $[0,1]$. Since $g_{n}(x)=g_{n}(1-x)$, we need only prove the second assertion on $\left[0, \frac{1}{2}\right]$. Now

$$
\frac{g_{n}^{\prime}(x)}{g_{n}(x)}=\frac{1}{x}-\frac{1}{n+1-x}-2 \sum_{k=1}^{n} \frac{x}{k^{2}-x^{2}},
$$

which is clearly decreasing. Thus for $x \in\left(0, \frac{1}{2}\right)$,

$$
\frac{g_{n}^{\prime}(x)}{g_{n}(x)}>2-\frac{2}{2 n+1}-\sum_{k=1}^{n} \frac{4}{4 k^{2}-1}=0 .
$$


Theorem 7.2. For each $r \in(0,1)$, let $f, g$ be defined on $[0,1]$, by $f(a)=$ $F\left(a-1,1-a ; 1 ; r^{2}\right)$ and $g(a)=F\left(a, 1-a ; 1 ; r^{2}\right)$.

(1) If $\frac{1}{2} \leq a<b \leq 1$, then all coefficients are positive in the Taylor series for $f(b)-f(a)$ in powers of $r^{2}$.

(2) If $0 \leq a<b \leq 1-1 / \sqrt{2}$, then all coefficients are negative in the Taylor series for $f(b)-f(a)-(b-a)(2-a-b) r^{2}$ in powers of $r^{2}$.

(3) If $0 \leq a<b \leq \frac{1}{2}$ (respectively, $\left.\frac{1}{2} \leq a<b \leq 1\right)$, then all coefficients are positive (respectively, negative) in the Taylor series for $g(b)-g(a)$ in powers of $r^{2}$.

Proof. (1) In this case,

$$
f(b)-f(a)=\sum_{n=1}^{\infty}[(1-b, n)(b-1, n)-(1-a, n)(a-1, n)] \frac{r^{2 n}}{(n !)^{2}} .
$$

Now $-(1-a, n)(a-1, n)-(-(1-b, n)(b-1, n))>0$, by Lemma 7.1.

(2) In this case, $1 \geq 1-a>1-b \geq 1 / \sqrt{2}$. Hence

$$
(1-b, n)(b-1, n)-(1-a, n)(a-1, n)<0,
$$

for all $n \geq 2$, by Lemma 7.1.

(3) We write

$$
g(b)-g(a)=\sum_{n=1}^{\infty}[(b, n)(1-b, n)-(a, n)(1-a, n)] \frac{r^{2 n}}{(n !)^{2}} .
$$

Now, $(b, n)(1-b, n)-(a, n)(1-a, n)$ is positive or negative according as $0 \leq a<b \leq \frac{1}{2}$, or $\frac{1}{2} \leq a<b \leq 1$, by Lemma 7.1.

Corollary 7.3. For each $r \in(0,1)$, the function

(1) $f(a) \equiv \mathcal{E}_{1-a}(r)$ is decreasing from $\left[0, \frac{1}{2}\right]$ onto $[\mathcal{E}(r), \pi / 2]$.

(2) $g(a) \equiv \mathcal{K}_{a}(r)$ is increasing from $\left[0, \frac{1}{2}\right]$ onto $[\pi / 2, \mathcal{K}(r)]$.

(3) $h(a) \equiv \mathcal{E}_{a}(r)$ is increasing from $\left[0, \frac{1}{2}\right]$ onto $\left[\pi r^{\prime 2} / 2, \mathcal{E}(r)\right]$.

Proof. Parts (1) and (2) follow from (1.9), (1.10), and Theorem 7.2. Next, by Theorem $4.1(4),(d / d r)\left(\mathcal{E}_{a}(r)-r^{\prime 2} \mathcal{K}_{a}(r)\right)=2 a r \mathcal{K}_{a}(r)$, which is increasing in $a$ by (2). Hence, if $0<a<b \leq \frac{1}{2}$,

$$
\int_{0}^{r} \frac{d}{d t}\left(\mathcal{E}_{a}(t)-t^{\prime 2} \mathcal{K}_{a}(t)\right) d t<\int_{0}^{r} \frac{d}{d t}\left(\mathcal{E}_{b}(t)-t^{\prime 2} \mathcal{K}_{b}(t)\right) d t
$$

so that

$$
\mathcal{E}_{a}(r)-r^{\prime 2} \mathcal{K}_{a}(r)<\mathcal{E}_{b}(r)-r^{\prime 2} \mathcal{K}_{b}(r)
$$

by Lemma $5.2(1)$. Thus

$$
\mathcal{E}_{a}(r)<\mathcal{E}_{b}(r)+r^{\prime 2}\left(\mathcal{K}_{a}(r)-\mathcal{K}_{b}(r)\right)<\mathcal{E}_{b}(r)
$$

by (2). 
Theorem 7.4. (1) For each fixed $r \in(0,1)$, the function $f(a, r) \equiv \mu_{a}(r)$ is decreasing in a from $\left(0, \frac{1}{2}\right]$ onto $[\mu(r), \infty)$.

(2) For each fixed $x \in(0, \infty)$, the function $g(a, x) \equiv \mu_{a}^{-1}(x)$ is decreasing in a from $\left(0, \frac{1}{2}\right]$ onto $\left[\mu^{-1}(x), 1\right)$.

(3) For each fixed $r \in(0,1)$ and $K \in(1, \infty)$, the function $h(a, r) \equiv \varphi_{K}^{a}(r)$ is decreasing in a from $\left(0, \frac{1}{2}\right]$ onto $\left[\varphi_{K}(r), 1\right)$. Moreover, the function $h_{1}(a, r) \equiv \varphi_{1 / K}^{a}(r)$ is increasing in a from $\left(0, \frac{1}{2}\right]$ onto $\left(0, \varphi_{1 / K}(r)\right]$.

Proof. (1) Part (1) was obtained in [QVu2, Theorem 1.22]. However, we give here a different proof for the monotoneity of $f$ in $a$. It follows from Theorem 4.1 (5) and Corollary 7.3 (2) that $d \mu_{a} / d r$ is increasing in $a$ on $\left(0, \frac{1}{2}\right]$. Hence, for $0<a<b \leq \frac{1}{2}$,

$$
\mu_{a}(1-)-\mu_{a}(r)=\int_{r}^{1} \frac{d \mu_{a}(t)}{d t} d t<\int_{r}^{1} \frac{d \mu_{b}(t)}{d t} d t=\mu_{b}(1-)-\mu_{b}(r) .
$$

This implies that $\mu_{a}(r)>\mu_{b}(r)$, since $\mu_{a}(1-)=0=\mu_{b}(1-)$.

(2) Let $r=g(a, x)=\mu_{a}^{-1}(x)$. Then $x=\mu_{a}(r)=f(a, r)$, and

$$
0=\frac{d x}{d a}=\frac{\partial f}{\partial a}+\frac{\partial f}{\partial r} \frac{d r}{d a} .
$$

Since $\partial f / \partial a<0$ by part (1) (see also [QVu2, 3.1]), it follows from Theorem 4.1 (5) that $d r / d a=-(\partial f / \partial a) /(\partial f / \partial r)<0$, and the monotoneity of $g$ in $a$ follows. Clearly, $g\left(\frac{1}{2}, x\right)=\mu^{-1}(x)$. Fix $x \in(0, \infty)$. Suppose $g(0+, x)=r_{0} \in$ $(0,1)$. Choose $\epsilon>0$ such that $\left(r_{0}-\epsilon, r_{0}+\epsilon\right) \subset(0,1)$. Then there exists $\delta \in(0,1)$ such that $g(a, x)<r_{0}+\epsilon$ for all $a \in(0, \delta)$. Hence $x=\mu_{a}(g(a, x))>$ $\mu_{a}\left(r_{0}+\epsilon\right)$. If we now let $a \rightarrow 0+$, we get $x=\infty$, a contradiction. Thus,

$$
g(0+, x)=1 \text {. }
$$

(3) Put $s=h(a, r), a_{n}=(a, n)(1-a, n)$, and $b_{n}=P(a, n)-P(a, 0)$ for $n=0,1,2, \ldots$, where $P(a, n)=\psi(a+n)-\psi(1-a+n)$, and let $Q(a, r)=$ $2 \mathcal{K}_{a}(r) / \pi=F\left(a, 1-a ; 1 ; r^{2}\right)$. Then, by (1.3), (1.5), and (1.2),

$$
\frac{Q\left(a, s^{\prime}\right)}{Q(a, s)}=\frac{1}{K} \frac{Q\left(a, r^{\prime}\right)}{Q(a, r)}
$$

and

$$
\frac{\partial Q(a, r)}{\partial r}=2 \sum_{n=1}^{\infty} \frac{n a_{n}}{(n !)^{2}} r^{2 n-1}, \quad \frac{\partial Q(a, r)}{\partial a}=\sum_{n=1}^{\infty} \frac{a_{n} b_{n}}{(n !)^{2}} r^{2 n}
$$

By (7.7), it follows from (7.6) and logarithmic differentiation with respect to $a$ that

$$
\begin{gathered}
\frac{1}{Q\left(a, s^{\prime}\right)}\left[\frac{\partial Q\left(a, s^{\prime}\right)}{\partial a}-\frac{s}{s^{\prime}} \frac{\partial Q\left(a, s^{\prime}\right)}{\partial s^{\prime}} \frac{d s}{d a}\right] \\
-\frac{1}{Q(a, s)}\left[\frac{\partial Q(a, s)}{\partial a}+\frac{\partial Q(a, s)}{\partial s} \frac{d s}{d a}\right]
\end{gathered}
$$




$$
=\frac{1}{Q\left(a, r^{\prime}\right)} \frac{\partial Q\left(a, r^{\prime}\right)}{\partial a}-\frac{1}{Q(a, r)} \frac{\partial Q(a, r)}{\partial a},
$$

and hence, by simplification,

$$
\begin{aligned}
& {\left[\frac{s}{s^{\prime} Q\left(a, s^{\prime}\right)} \frac{\partial Q\left(a, s^{\prime}\right)}{\partial s^{\prime}}+\frac{1}{Q(a, s)} \frac{\partial Q(a, s)}{\partial s}\right] \frac{d s}{d a}} \\
& =\left[Q_{1}\left(a, s^{\prime}\right)-Q_{1}\left(a, r^{\prime}\right)\right]+\left[Q_{1}(a, r)-Q_{1}(a, s)\right],
\end{aligned}
$$

where

$$
Q_{1}(a, x)=\frac{1}{Q(a, x)} \frac{\partial Q(a, x)}{\partial a}=\frac{\sum_{n=1}^{\infty} \alpha_{n} x^{2 n}}{\sum_{n=0}^{\infty} \beta_{n} x^{2 n}}=\frac{\sum_{n=0}^{\infty} \alpha_{n} x^{2 n}}{\sum_{n=0}^{\infty} \beta_{n} x^{2 n}},
$$

and where $\alpha_{n}=a_{n} b_{n}(n !)^{-2}$ and $\beta_{n}=a_{n}(n !)^{-2}$. Since

$$
\frac{\alpha_{n}}{\beta_{n}}=b_{n}=\psi(1-a)-\psi(a)-(1-2 a) \sum_{k=0}^{\infty} \frac{1}{(a+n+k)(1-a+n+k)}
$$

(cf. [Ah, p. 198]), which is clearly increasing in $n$, it follows from [PV, Lemma 2.1] that $Q_{1}(a, x)$ is increasing in $x$ on $(0,1)$. Since $\partial Q(a, x) / \partial x>0$ by Theorem 4.1 (1), and since $s>r(s<r$, respectively) for $K>1(K<1$, respectively), the monotoneity properties of $h$ and $h_{1}$ in $a$ follow from (7.8).

In case $K=1$, we have $\varphi_{K}^{a}(r)=r$ for all $a \in(0,1)$. Next, supppose $K>1$. Take $0<L=\varphi_{K}^{0+}(r)<1$, and choose $\epsilon>0$ such that $(L-\epsilon, L+$ $\epsilon) \subset(0,1)$. Then there exists $\delta \in(0,1)$ such that $a \in(0, \delta)$ implies that $\varphi_{K}^{a}(r)<L+\epsilon=t$, say. Hence, $1 / K>\mu_{a}(t) / \mu_{a}(r)$. If we let $a \rightarrow 0+$, we get $1 / K \geq 1$, a contradiction. Hence $L=1$. Finally, if $0<K<1$, then the assertion follows from the result for the case $K>1$ and Lemma 6.1 (1).

Acknowledgments. This work was carried out during the visits of the third and fourth authors to Michigan State University, USA, and that of the third author to the University of Helsinki, Finland, and the Hangzhou Institute of Electronics Engineering, China. The authors would like to thank these institutions and the University of Auckland, the Academy of Finland, and the Finnish Mathematical Association for hospitality and support.

We are indebted to R. Askey for discussions and advice concerning Section 3, and to F. Garvan for bringing reference [Mi] to our attention.

\section{References}

[AS] M. Abramowitz and I.A. Stegun, editors, Handbook of Mathematical Functions with Formulas, Graphs and Mathematical Tables, Dover, New York, 1965.

[Ah] L.V. Ahlfors, Complex Analysis, 2nd ed., McGraw-Hill, New York, 1966.

[ABRVV] G.D. Anderson, R.W. Barnard, K.C. Richards, M.K. Vamanamurthy and M. Vuorinen, Inequalities for zero-balanced hypergeometric functions, Trans. Amer. Math. Soc., 347 (1995), 1713-1723. 
[AVV] G.D. Anderson, M.K. Vamanamurthy and M. Vuorinen, Conformal Invariants, Inequalities, and Quasiconformal Maps, John Wiley \& Sons, New York, 1997.

[Ask] R. Askey, Ramanujan and hypergeometric and basic hypergeometric series, Ramanujan Internat. Symposium on Analysis, December 26-28, 1987, ed. by N.K. Thakare, 1-83, Pune, India, Russian Math. Surveys, 451 (1990), 37-86.

[BPV] R. Balasubramanian, S. Ponnusamy and M. Vuorinen, Functional inequalities for the quotients of hypergeometric functions, J. Math. Anal. Appl., 218 (1998), 256-268.

[Ba] Bateman Manuscript Project (ed. A. Erdélyi, W. Magnus, F. Oberhettinger and F.G. Tricomi), Higher Transcendental Functions, Vol. I, McGraw-Hill, New York, 1953.

[Be1] B.C. Berndt, Ramanujan's Notebooks, Vol. I, Springer-Verlag, BerlinHeidelberg-New York, 1985.

[Be2] _ Ramanujan's Notebooks, Vol. II, Springer-Verlag, Berlin-HeidelbergNew York, 1989.

[Be3] _ Ramanujan's Notebooks, Vol. III, Springer-Verlag, Berlin-HeidelbergNew York, 1991.

[BBG] B.C. Berndt, S. Bhargava and F.G. Garvan, Ramanujan's theories of elliptic functions to alternative bases, Trans. Amer. Math. Soc., 347 (1995), 4163-4244.

[BB1] J.M. Borwein and P.B. Borwein, Pi and the AGM, John Wiley \& Sons, New York, 1987.

[BB2] A cubic counterpart of Jacobi's identity and the AGM, Trans. Amer. Math. Soc., 323 (1991), 691-701.

[Bo] F. Bowman, Introduction to Elliptic Functions with Applications, Dover, New York, 1961.

[BF] P.F. Byrd and M.D. Friedman, Handbook of Elliptic Integrals for Engineers and Physicists, 2nd ed., Die Grundlehren Math. Wiss., 67, Springer-Verlag, Berlin-Göttingen-Heidelberg-New York, 1971.

[C] I. Chavel, Riemannian Geometry - A Modern Introduction, Cambridge Tracts in Math., 108, Cambridge Univ. Press, 1993.

[Di] A.L. Dixon, Generalizations of Legendre's formula $K E^{\prime}-(K-E) K^{\prime}=\frac{1}{2} \pi$, Proc. London Math. Soc., 3(2) (1905), 206-224.

[Du] P.L. Duren, The Legendre relation for elliptic integrals, in Paul Halmos: Celebrating 50 Years of Mathematics, ed. by J.H. Ewing and F.W. Gehring, Springer-Verlag, New York, (1991), 305-315.

[El] E.B. Elliott, A formula including Legendre's $E K^{\prime}+K E^{\prime}-K K^{\prime}=\frac{1}{2} \pi$, Messenger of Math., 33 (1904), 31-40.

[Ga] F.G. Garvan, Ramanujan's theories of elliptic functions to alternative bases a symbolic excursion, J. Symbolic Comput., 20 (1995), 517-536.

[LV] O. Lehto and K.I. Virtanen, Quasiconformal Mappings in the Plane, 2nd ed., Die Grundlehren der Math. Wiss., 126, Springer-Verlag, New York-Berlin, 1973.

[Mark] A.I. Markushevich, Theory of Functions of a Complex Variable, Vol. II, Prentice-Hall, Englewood Cliffs, NJ, 1965. 
[Mart] G.J. Martin, The distortion theorem for quasiconformal mappings, Schottky's theorem and holomorphic motions, Proc. Amer. Math. Soc., 125 (1997), 10951103.

[Mi] W. Miller, Jr., Symmetry and separation of variables, With a foreword by Richard Askey; Encyclopedia of Mathematics and its Applications, Vol. 4, Reading, Massachusetts, Addison-Wesley Publishing Company, XXX (1977).

[PV] S. Ponnusamy and M. Vuorinen, Asymptotic expansions and inequalities for hypergeometric functions, Mathematika, 44 (1997), 278-301.

[QVu1] S.-L. Qiu and M. Vuorinen, Landen inequalities for hypergeometric functions, Nagoya Math. J., 154 (1999), 31-56.

[QVu2] _ Duplication inequalities for the ratios of hypergeometric functions, Forum Math., 11 (1999), to appear.

[QVu3] , Infinite products and the normalized quotients of hypergeometric functions, SIAM J. Math. Anal., 30 (1999), 1057-1075.

[R] E.D. Rainville, Special Functions, MacMillan, New York, 1960.

[Var] V.S. Varadarajan, Linear meromorphic differential equations: A modern point of view, Bull. Amer. Math. Soc., 33 (1996), 1-42.

[WW] E.T. Whittaker and G.N. Watson, A Course of Modern Analysis, 4th ed., Cambridge Univ. Press, London, 1958.

Received April 23, 1998.

Michigan State University

EAst LANSING, MI 48824

E-mail address: anderson@math.msu.edu

Hangzhou Institute of Electronics Engineering

HANGZHOU 310037

P.R. CHINA

University of Auckland

Auckland

NEW ZEALAND

E-mail address: vamanamu@math.auckland.nz

UNIVERSITY OF HELSINKI

FIN-00014

FINLAND

E-mail address: vuorinen@csc.fi 